\title{
ON THE TRIANGLE REMOVAL LEMMA FOR SUBGRAPHS OF SPARSE PSEUDORANDOM GRAPHS
}

\author{
YOSHIHARU KOHAYAKAWA, VOJTĚCH RÖDL, MATHIAS SCHACHT, \\ AND JOZEF SKOKAN \\ Dedicated to Endre Szemerédi on the occasion of his 70 th birthday
}

\begin{abstract}
We study an extension of the triangle removal lemma of Ruzsa and Szemerédi [Triple systems with no six points carrying three triangles, Combinatorics (Proc. Fifth Hungarian Colloq., Keszthely, 1976), Vol. II, NorthHolland, Amsterdam, 1978, pp. 939-945], which gave rise to a purely combinatorial proof of the fact that sets of integers of positive upper density contain three-term arithmetic progressions, a result first proved by Roth [On certain sets of integers, J. London Math. Soc. 28 (1953), 104-109].

We obtain a generalization of the triangle removal lemma for subgraphs of sparse pseudorandom graphs and deduce the following version of Roth's theorem, which applies to sparse sets of integers: If $A \subseteq[n]=\{1, \ldots, n\}$ has the property that all non-trivial Fourier coefficients $\lambda$ of the indicator function $\mathbb{1}_{A}:[n] \rightarrow\{0,1\}$ satisfy $|\lambda|=o\left(|A|^{3} / n^{2}\right)$, then any subset $B \subseteq A$ that contains no three-term arithmetic progression satisfies $|B|=o(|A|)$.
\end{abstract}

\section{INTRODUCTION}

In the mid-seventies Ruzsa and Szemerédi [25] solved a problem of Brown, Erdős, and Sós [5] and established the so-called triangle removal lemma. Loosely speaking, the triangle removal lemma asserts that every graph which does not contain many triangles can be made triangle-free by removing only a few edges. More precisely, for a positive integer $n$ we denote by $K_{n}$ the complete graph with vertex set $[n]=$ $\{1, \ldots, n\}$. For two graphs $F$ and $H$ we say $H$ is $F$-free, if $H$ does not contain a (not necessarily induced) copy of $F$. For a graph $G=(V, E)$ we denote the minimum number of edges that meet any triangle in $G$ by

$$
\tau_{3}(G)=\min \left\{\left|E^{\prime}\right|: E^{\prime} \subseteq E \text { and } H=\left(V, E \backslash E^{\prime}\right) \text { is } K_{3} \text {-free }\right\} .
$$

Theorem 1 (Ruzsa \& Szemerédi). For every $\delta>0$ there exist $c>0$ and $n_{0}$ such that every graph $G=(V, E)$ with $|V|=n \geq n_{0}$ that contains at most $c\left(\begin{array}{l}n \\ 3\end{array}\right)$ copies of $K_{3}$ satisfies $\tau_{3}(G) \leq \delta\left(\begin{array}{c}n \\ 2\end{array}\right)$.

Theorem 1 stimulated a great deal of research and several generalizations for graphs and hypergraphs are now known (see, e.g., [23] and the references therein and [10] for a new proof of Theorem 1). Moreover, it was already shown in [25] that

Date: May 10, 2010.

The collaboration of the first and the third author was supported by a CAPES-DAAD collaboration grant.

The first author was partially supported by FAPESP and CNPq through a Temático-ProNEx project (Proc. FAPESP 2003/09925-5) and by CNPq (Proc. 308509/2007-2, 485671/2007-7 and $486124 / 2007-0)$. The second author was supported by the NSF grant DMS 0800070. 
Theorem 1 yields a new proof of Roth's famous theorem on arithmetic progressions of length three [24] (see [3] for the best known quantitative bound). We say a set of integers is $A P_{3}-f r e e$, if it does not contain a non-trivial three-term arithmetic progression, i.e., three distinct elements $x, y, z$ such that $x+z=2 y$. For a finite set of integers $A$ we denote the maximum size of an $\mathrm{AP}_{3}$-free subset of $A$ by

$$
r_{3}(A)=\max \left\{|B|: B \subseteq A \text { and } B \text { is } \mathrm{AP}_{3} \text {-free }\right\}
$$

and we simply write $r_{3}(n)$ for $r_{3}([n])$.

Theorem 2 (Roth). For every $\delta>0$ there exists $n_{0}$ such that for all $n \geq n_{0}$ we have $r_{3}(n) \leq \delta n$.

Sparse versions of Theorems 1 and 2 were studied in the context of random discrete structures. In particular, in [16] it was shown that, for any $\delta>0$, with high probability a random subsets $A \subseteq[n]$ with $|A|=C_{\delta} n^{1 / 2}$ satisfies $r_{3}(A) \leq \delta|A|$, where $C_{\delta}$ depends only on $\delta$ (see [26] for generalizations of this result to arithmetic progressions of length $k>3$ and see [21] for an alternative proof for the case $k=$ $3)$. The techniques developed in [16] can be used to obtain a similar extension of Theorem 1 for subgraphs of random graphs. This extension asserts that for every fixed $\delta>0$ there exist constants $C_{\delta}$ and $c_{\delta}>0$ such that if $p \geq C_{\delta} n^{-1 / 2}$ then a random graph $\Gamma \in G(n, p)$ satisfies with high probability the following: every subgraph $G \subseteq \Gamma$ that contains at most $c_{\delta} p^{3}\left(\begin{array}{l}n \\ 3\end{array}\right)$ copies of $K_{3}$ satisfies $\tau_{3}(G) \leq \delta p\left(\begin{array}{l}n \\ 2\end{array}\right)$. Note that Theorem 1 corresponds to the case $p=1$ and Theorem 1 implies such a result for any constant $p>0$.

Another example of a relative version of Roth's theorem appears in the work of Green [12] who showed that, for the set of primes $\mathbb{P}$, we have $r_{3}(\mathbb{P} \cap[n])=o(|\mathbb{P} \cap[n]|)$, i.e., any relatively dense subset of the primes contains an arithmetic progression of length three (see [14] for longer arithmetic progressions).

We obtain extensions of Theorems 1 and 2 for subgraphs of sparse pseudorandom graphs (see Theorem 3) and for subsets of sparse pseudorandom subsets of $[n]$ (see Theorem 4). Our proof is based on the sparse regularity lemma $[15,17]$ and the main technical result presented here (see part $(a)$ of Lemma 9 below).

\section{NEW RESUlts}

Next we define the notions of pseudorandomness considered here. Roughly speaking, pseudorandom discrete structures "imitate" a truly random object of the same density. The systematic study of pseudorandom graphs was initiated by Thomason [28, 29] and continued by Chung, Graham, and Wilson [7] and we will use a related concept here. In fact, Chung and Graham obtained several generalizations of those results for other discrete structures and one of the properties of pseudorandom subsets of $\mathbb{Z} / n \mathbb{Z}$ studied in [6] is related to our concept of pseudorandom subsets of $[n]$.

2.1. The triangle removal lemma for pseudorandom graphs. We say a graph $\Gamma=(V, E)$ is $(p, \beta)$-bijumbled for $p$ and $\beta>0$, if all subsets $X, Y \subseteq V$ satisfy

$$
\left|e_{\Gamma}(X, Y)-p\right| X|| Y|| \leq \beta \sqrt{|X||Y|},
$$

where $e_{\Gamma}(X, Y)=|\{(x, y) \in X \times Y:\{x, y\} \in E(\Gamma)\}|$ denotes the number of edges of $\Gamma$ with one endvertex in $X$ and the other endvertex in $Y$, where edges contained in $X \cap Y$ are counted twice. 
It follows from Chernoff's inequality that the binomial random graph $G(n, p)$ is with high probability $(p, \beta)$-bijumbled for $\beta=C \sqrt{p n}$ for some sufficiently large constant $C>1$, if $p n-3 \log n \rightarrow \infty$. On the other hand, it follows from the work of Erdős and Spencer [9] (see also [8]) that for every $(p, \beta)$-bijumbled $n$-vertex graph we have $\beta=\Omega(\sqrt{p(1-p) n})$ as long as $p(1-p)>1 / n$.

A well known explicit class of $(p, \beta)$-bijumbled graphs are the so-called $(n, D, \lambda)$ graphs with $D=p n$ and $\lambda=\beta$. An $(n, D, \lambda)$-graph $\Gamma$ is a $D$-regular $n$-vertex graph which satisfies $\lambda \geq\left\{\lambda_{2},\left|\lambda_{n}\right|\right\}$, where $D=\lambda_{1} \geq \lambda_{2} \geq \cdots \geq \lambda_{n}$ are the eigenvalues of the adjacency matrix of $\Gamma$ (see [2, Chapter 9] and [18] for more details).

Our first result asserts that Theorem 1 can be extended to subgraphs $G \subseteq \Gamma$ of $(p, \beta)$-bijumbled $n$-vertex graphs $\Gamma$, if $\beta \leq \gamma p^{3} n$ for a sufficiently small $\gamma>0$.

Theorem 3. For every $\delta>0$ there exist $c>0, \gamma>0$, and $n_{0}$ such that the following holds.

Suppose $\Gamma=\left(V, E_{\Gamma}\right)$ is a $\left(p, \gamma p^{3} n\right)$-bijumbled graph with $n$ vertices and $p \geq 1 / \sqrt{n}$ and let $G=\left(V, E_{G}\right) \subseteq \Gamma$ be a (not necessarily induced) subgraph of $\Gamma$. If $G$ contains at most $\mathrm{cp}^{3}\left(\begin{array}{l}n \\ 3\end{array}\right)$ copies of $K_{3}$, then $\tau_{3}(G) \leq \delta e(\Gamma)$.

We remark the following:

(I) It is easy to show that for sufficiently small $\gamma_{\xi}>0$ every $\left(p, \gamma p^{2} n\right)$-bijumbled $n$-vertex graph $\Gamma$ contains $(1 \pm \xi) p^{3}\left(\begin{array}{l}n \\ 3\end{array}\right)$ copies of $K_{3}$. Consequently, Theorem 3 asserts that if a subgraph $G \subseteq \Gamma$ contains only a small fraction of the triangles of the sufficiently pseudorandom host graph $\Gamma$, then $G$ can be made triangle-free by removing a small fraction of the edges of $\Gamma$. In Theorem 1 the complete graph $K_{n}$ plays the same rôle as $\Gamma$.

(II) Note that Theorem 3 applies only to $(p, \beta)$-bijumbled $n$-vertex graphs $\Gamma$ with $\beta=\gamma p^{3} n$ for sufficiently small $\gamma$. Since $\beta=\Omega(\sqrt{p n})$ for every graph with $p(1-p) \geq 1 / n$, it follows that Theorem 3 only applies to graphs $\Gamma$ of density $p=p(n)=\Omega\left(n^{-1 / 5}\right)$.

We believe Theorem 3 is also true for $(p, \beta)$-bijumbled $n$-vertex graphs $\Gamma$ with $\beta=\gamma p^{2} n$, which would allow us to consider graphs of density $p \geq$ $C n^{-1 / 3}$. On the other hand, Alon [1] constructed a $(p, 19 \sqrt{p n})$-bijumbled $n$-vertex graph $\Gamma$ with $p=n^{-1 / 3} / 4$ which is $K_{3}$-free. This indicates that such a strengthening Theorem 3 would be "best possible."

2.2. Roth's theorem for pseudorandom sets. For $A \subseteq[n]$ we consider the discrete Fourier coefficients $\lambda_{0}(A), \ldots, \lambda_{n-1}(A) \in \mathbb{C}$ of $A$ defined by

$$
\lambda_{k}(A)=\sum_{a \in A} \exp \left(\frac{2 \pi \mathrm{i}}{n} \cdot k a\right) .
$$

Clearly, $\lambda_{0}(A)=|A|$ and we set

$$
\lambda(A)=\max \left\{\left|\lambda_{k}(A)\right|: k=1, \ldots, n-1\right\} .
$$

Theorem 4 extends Theorem 2 to sparse sets $A \subseteq[n]$ for which $\lambda(A)$ is "small." A somewhat related result appears in the work of Green and Tao [13, Proposition 5.1].

Theorem 4. For every $\delta>0$ there exist $\gamma>0$ and $n_{0}$ such that for every odd $n \geq n_{0}$ and every $A \subseteq[n]$ with $\lambda(A) \leq \gamma|A|^{3} / n^{2}$ we have $r_{3}(A) \leq \delta|A|$. 
Very roughly speaking, the condition on $A$ in Theorem 4 asserts that a certain Cayley-type graph $\Gamma(A)$ generated by $A$ on the vertex set $[n]$ is an $\left(n,|A|, \gamma|A|^{3} / n^{2}\right)$ graph and, therefore, a $\left(p, \gamma p^{3} n\right)$-bijumbled graph for $p=|A| / n$. This allows us to apply similar techniques as in the proof of Theorem 3.

In view of Remark (II) after Theorem 3 we believe that the condition on $\lambda(A)$ in Theorem 4 can be relaxed to $\lambda(A) \leq \gamma|A|^{2} / n$.

2.3. Key technical result. The main technical result behind the proofs of Theorems 3 and 4, namely, Lemma 9, concerns the theory of pseudorandom properties, which are asserted by the sparse regularity lemma. For the statement of Lemma 9 we require a few definitions.

Definition 5 (DISC). Let $G=(V, E)$ be a graph and let $X, Y \subseteq V$ be disjoint. We say $(X, Y)$ satisfies the discrepancy condition $\operatorname{DISC}(q, p, \varepsilon)$ in $G$ for some $q, p$, and $\varepsilon>0$ if the following holds:

$$
\left|e_{G}\left(X^{\prime}, Y^{\prime}\right)-q\right| X^{\prime}|| Y^{\prime}|| \leq \varepsilon p|X||Y| \quad \text { for all } X^{\prime} \subseteq X \text { and } Y^{\prime} \subseteq Y \text {. }
$$

As a shorthand, we shall sometimes say $(X, Y)_{G}$ satisfies $\operatorname{DISC}(q, p, \varepsilon)$.

Note that Szemerédi's regularity lemma [27] asserts that the vertex set of every $n$-vertex graph $G$ can be partitioned into a bounded number of classes $V_{1} \dot{\cup} \ldots \dot{U} V_{t}$ in such a way that most pairs $\left(V_{i}, V_{j}\right)_{G}$ satisfy $\operatorname{DISC}\left(q_{i j}, p, \varepsilon\right)$, where

$$
q_{i j}=d_{G}\left(V_{i}, V_{j}\right)=\frac{e_{G}\left(V_{i}, V_{j}\right)}{\left|V_{i}\right|\left|V_{j}\right|} \text { and } p=1 .
$$

Since $\operatorname{DISC}(q, p, \varepsilon)$ only gives useful information on the edge distribution of $(X, Y)_{G}$ if $q>\varepsilon p$, Szemerédi's regularity lemma is mainly suited for applications to "dense" graphs $G$ with $\Omega\left(|V(G)|^{2}\right)$ edges. The first two authors observed that Szemerédi's regularity lemma can be extended to subgraphs $G$ of sparse graphs $\Gamma$, provided the host graph $\Gamma$ does not contain "dense spots." In this context $p$ can be chosen to be $e(\Gamma) /\left(\begin{array}{l}n \\ 2\end{array}\right)$, i.e., it can tend to 0 as $n$ tends to infinity. For example, $(p, \beta)$ bijumbled graphs $\Gamma$ do not contain "dense spots" as long as $\beta \leq \gamma p n$ for some small constant $\gamma>0$. Consequently, the sparse regularity lemma is applicable to the graphs $G \subseteq \Gamma$ satisfying the assumptions of Theorem 3. For the proof of Theorem 3 we will need a corresponding "counting lemma" for triangles in tripartite graph with all three induced bipartite graphs satisfying DISC (see Lemma 11 below). For that we will show that pairs $(X, Y)_{G}$ satisfying DISC will also satisfy the so called pair condition. For (not necessarily distinct) vertices $x$ and $x^{\prime} \in V$ and a subset $Y \subseteq V$ we write $\operatorname{deg}_{G}(x, Y)$ for the number of neighbors of $x$ in $Y$ and we write $\operatorname{deg}_{G}\left(x, x^{\prime}, Y\right)$ for the number of joint neighbors of $x$ and $x^{\prime}$ in $Y$. Moreover, we denote the sets of such neighbors by $N_{G}(x, Y)$ and $N_{G}\left(x, x^{\prime}, Y\right)$

Definition 6 (PAIR). Let $G=(V, E)$ be a graph and let $X, Y \subseteq V$ be disjoint. $W e$ say $(X, Y)$ satisfies the pair condition $\operatorname{PAIR}(q, p, \delta)$ in $G$ for some $q, p$, and $\delta>0$ if the following holds:

$$
\begin{gathered}
\sum_{x \in X}\left|\operatorname{deg}_{G}(x, Y)-q\right| Y|| \leq \delta p|X||Y|, \\
\sum_{x \in X} \sum_{x^{\prime} \in X}\left|\operatorname{deg}_{G}\left(x, x^{\prime}, Y\right)-q^{2}\right| Y|| \leq \delta p^{2}|X|^{2}|Y| .
\end{gathered}
$$

As a shorthand, we shall sometimes say $(X, Y)_{G}$ satisfies $\operatorname{PAIR}(q, p, \delta)$. 
It is well known (see, e.g., [7]) that DISC and PAIR are equivalent in the dense case, i.e., for $p=1$.

Theorem 7 (DISC $\Leftrightarrow$ PAIR (dense case)). For every $\alpha>0$ and $\delta>0$ there exist $\varepsilon>0$ and $n_{0}$ such that the following holds.

Suppose $G=(V, E)$ is a graph and $X, Y \subseteq V$ are disjoint sets with $|X|,|Y| \geq n_{0}$. Then the following statements hold:

(a) if $(X, Y)_{G}$ satisfies $\operatorname{DISC}(q, 1, \varepsilon)$ for some $q$ with $\alpha \leq q \leq 1$, then it also satisfies $\operatorname{PAIR}(q, 1, \delta)$;

(b) if $(X, Y)_{G}$ satisfies $\operatorname{PAIR}(q, 1, \varepsilon)$ for some $q$ with $\alpha \leq q \leq 1$, then it also satisfies $\operatorname{DISC}(q, 1, \delta)$.

The key technical lemma presented here asserts that an analogue of Theorem 7 is true for subgraphs of $(p, \beta)$-bijumbled graphs $\Gamma$ if $\beta$ is sufficiently small. In fact, we can slightly relax the bijumbledness condition and consider a partite version instead. For simplicity we call this property jumbledness.

Definition 8 (jumbledness (partite version)). Let $\Gamma=\left(U \dot{U} V, E_{\Gamma}\right)$ be a bipartite graph and $q, \beta>0$ be real numbers. We say $\Gamma$ is $(q, \beta)$-jumbled if

$$
\left|e_{\Gamma}(X, Y)-q\right| X|| Y|| \leq \beta \sqrt{|X||Y|} \text { for all } X \subseteq U \text { and } Y \subseteq V .
$$

Moreover, we say a $k$-partite graph $\Gamma=\left(V_{1} \dot{\cup} \ldots \dot{U} V_{k}, E_{\Gamma}\right)$ with $k \geq 2$ is $(q, \beta)$ jumbled if all induced bipartite subgraphs $\Gamma\left[V_{i} \dot{\cup} V_{j}\right]$ for $1 \leq i<j \leq k$ are $(q, \beta)$ jumbled.

Note that both discrepancy and jumbledness are two measures of pseudorandomness for graphs.

In the context of this paper discrepancy $(\operatorname{DISC}(q, p, \varepsilon)$ where $\varepsilon>0$ and the ratio $q / p$ are constants independent of the size of the graph) is a property of subgraphs of $G$ that can be obtained by an application of the (sparse) regularity lemma.

On the other hand, jumbledness will be a property imposed on the host graph $\Gamma \supseteq G$. Typically the jumbledness assumption is stronger and cannot be ensured by an application of the regularity lemma.

Lemma 9 (DISC $\Leftrightarrow$ PAIR for subgraphs of jumbled graphs). For every $\alpha \in(0,1]$ and $\delta>0$ there exists $\varepsilon>0$ such that for every $\eta>0$ there exist $\gamma>0$ and $n_{0}$ such that for every $n \geq n_{0}$ the following holds.

Suppose

(i) $\Gamma=\left(U \dot{U} V, E_{\Gamma}\right)$ is a bipartite $(p, \beta)$-jumbled graph with $|U|,|V| \geq n$ and $p \geq 1 / \sqrt{n}$,

(ii) $G=\left(U \dot{U} V, E_{G}\right)$ is a subgraph of $\Gamma$, and

(iii) $X \subseteq U$ and $Y \subseteq V$ with $|X|,|Y| \geq \eta n$.

Then the following statements hold:

(a) if $\beta \leq \gamma p^{2} n$ and $(X, Y)_{G}$ satisfies $\operatorname{DISC}(q, p, \varepsilon)$ for some $q$ with $\alpha p \leq q \leq p$, then $(X, Y)_{G}$ satisfies $\operatorname{PAIR}(q, p, \delta)$;

(b) if $\beta \leq \gamma p^{3 / 2} n$ and $(X, Y)_{G}$ satisfies $\operatorname{PAIR}(q, p, \varepsilon)$ for some $q$ with $\alpha p \leq q \leq$ $p$, then $(X, Y)_{G}$ satisfies $\operatorname{DISC}(q, p, \delta)$.

The main part of this paper is devoted to the proof of implication $(a)$ of Lemma 9. The proofs of Theorems 3 and 4 rely on this implication. On the other hand, the proof of implication $(b)$ of the lemma can be established along the lines of the 
argument for the dense case (Theorem $7(b)$ ). We include the proof of part $(b)$ of Lemma 9 for completeness (see Section 5.1). We note that the jumbledness assumption on the host graph $\Gamma$ in part $(b)$ is less restrictive than in part $(a)$. We believe that the assumption for implication $(a)$ can be weakened (see Section 6)

Organization. In Section 3 we deduce Theorem 3 from Lemma 9(a) and the sparse regularity lemma. Section 4 is devoted to the proof of Theorem 4, which is based on a tripartite variant of Theorem 3 (see Theorem 10 below). The proof of the key technical result, Lemma 9, is deferred to Section 5 .

\section{The Ruzsa-Szemerédi theOrem for PSEUdorandom Graphs}

The proof presented here follows the standard proof of the Ruzsa-Szemerédi theorem, which is based on Szemerédi's regularity lemma and the so-called triangle counting lemma. In fact, the proof of Theorem 3 is based on the sparse regularity lemma $[15,17]$ and an appropriate triangle counting lemma for subgraphs of jumbled graphs (see Lemma 11 below), which we deduce from part ( $a$ ) of Lemma 9. First we state a tripartite version of Theorem 3 , which will be convenient for the proof of Theorem 4 .

3.1. Tripartite version of Theorem 3. We shall show that Theorem 3 easily follows from the following tripartite version.

Theorem 10 (tripartite version of Theorem 3). For every $\delta>0$ there exist $c>0$, $\gamma>0$, and $n_{0}$ such that for every $n \geq n_{0}$ the following holds.

Suppose $\Gamma=\left(V_{1} \dot{\cup} V_{2} \cup V_{3}, E_{\Gamma}\right)$ is a tripartite $\left(p, \gamma p^{3} n\right)$-jumbled graph with $\left|V_{i}\right| \geq n$

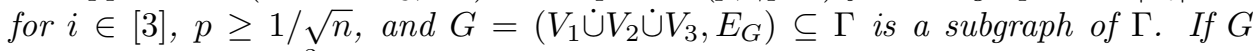
contains at most $c p^{3}\left|V_{1}\right|\left|V_{2}\right|\left|V_{3}\right|$ copies of $K_{3}$, then $\tau_{3}(G) \leq \delta e(\Gamma)$.

Proof: Theorem $10 \Rightarrow$ Theorem 3. For any given $\delta>0$ we set $\delta^{\prime}=\delta / 6$ and let $c^{\prime}$ and $\gamma^{\prime}>0$ be given by Theorem 10. For Theorem 3 we then set $c=c^{\prime}$ and $\gamma=\gamma^{\prime}$. Finally let $n$ be sufficiently large and $p \geq 1 / \sqrt{n}$.

Let $\Gamma=\left(V, E_{\Gamma}\right)$ be a $\left(p, \gamma p^{3} n\right)$-bijumbled graph and let $G=\left(V, E_{G}\right)$ be a subgraph of $\Gamma$ which contains at most $c p^{3}\left(\begin{array}{l}n \\ 3\end{array}\right)$ copies of $K_{3}$.

We consider tripartite graphs $\Gamma^{\prime}=\left(V^{\prime}, E_{\Gamma^{\prime}}\right) \supseteq G^{\prime}=\left(V^{\prime}, E_{G^{\prime}}\right)$ defined as follows

$$
V^{\prime}=V \times[3]
$$

and

$$
\begin{aligned}
& E_{\Gamma^{\prime}}=\left\{\{(u, i),(v, j)\}:\{u, v\} \in E_{\Gamma} \text { and } 1 \leq i<j \leq 3\right\}, \\
& E_{G^{\prime}}=\left\{\{(u, i),(v, j)\}:\{u, v\} \in E_{G} \text { and } 1 \leq i<j \leq 3\right\} .
\end{aligned}
$$

It follows from the definition of $G^{\prime}$ and $\Gamma^{\prime}$ that $e\left(\Gamma^{\prime}\right)=6 e(\Gamma),\left|V^{\prime}\right|=3 n$, and the assumptions on $\Gamma$ and $G$ yield

(a) $\Gamma^{\prime}$ is a tripartite $\left(p, \gamma p^{3} n\right)$-jumbled graph and

(b) $G^{\prime}$ contains at most $6 c p^{3}\left(\begin{array}{l}n \\ 3\end{array}\right) \leq c^{\prime} p^{3} n^{3}$ triangles.

Consequently, $\Gamma^{\prime}$ and $G^{\prime}$ satisfy the assumptions of Theorem 10 and, hence, there exists a set of edges $X^{\prime} \subseteq E_{G^{\prime}}$ such that $H^{\prime}=\left(V^{\prime}, E_{G^{\prime}} \backslash X\right)$ is $K_{3}$-free and

$$
\left|X^{\prime}\right| \leq \delta^{\prime} e\left(\Gamma^{\prime}\right)=\delta e(\Gamma) .
$$

We consider the set of edges $X$ which is the "pullback" of $X^{\prime}$ in $G$, i.e.,

$$
X=\left\{\{u, v\}:\{(u, i),(v, j)\} \in X^{\prime} \text { for some } 1 \leq i<j \leq 3\right\} .
$$


Clearly $|X| \leq\left|X^{\prime}\right| \leq \delta e(\Gamma)$ and it is easy to check that $H=\left(V, E_{G} \backslash X\right)$ is $K_{3}$-free. Therefore, $\tau_{3}(G) \leq \delta e(\Gamma)$.

3.2. Triangle counting lemma for subgraphs of tripartite jumbled graphs. The proof of Theorem 10 outlined here follows the lines of the standard proof of Theorem 1, which is based on Szemerédi's regularity lemma and a corresponding triangle counting lemma. In fact the sparse version of the regularity lemma applies to subgraphs of $\left(p, \gamma p^{3} n\right)$-jumbled tripartite graphs and below we state an appropriate triangle counting lemma for subgraphs of jumbled graphs.

Lemma 11. For every $\alpha \in(0,1]$ and $\xi>0$ there exists $\varepsilon>0$ such that for all $\eta>0$ there exist $\gamma>0$ and $n_{0}$ such that for every $n \geq n_{0}$ the following holds.

Suppose

(i) $\Gamma=\left(V_{1} \dot{\cup} V_{2} \dot{\cup} V_{3}, E_{\Gamma}\right)$ is a tripartite $\left(p, \gamma p^{3} n\right)$-jumbled graph with $\left|V_{i}\right| \geq n$ for $i \in[3]$ and $p \geq 1 / \sqrt{n}$,

(ii) $G=\left(V_{1} \cup V_{2} \cup \dot{\cup} V_{3}, E_{G}\right) \subseteq \Gamma$ is a subgraph of $\Gamma$, and

(iii) $U_{i} \subseteq V_{i}$ with $\left|U_{i}\right| \geq \eta n$ for $i \in[3]$.

If $e_{G}\left(U_{2}, U_{3}\right)=q_{23}\left|U_{2}\right|\left|U_{3}\right|$ for some $q_{23}$ with $\alpha p \leq q_{23} \leq p$ and for $j=2$ and 3 the pair $\left(U_{1}, U_{j}\right)_{G}$ satisfies $\operatorname{DISC}\left(q_{1, j}, p, \varepsilon\right)$ for some $q_{1, j}$ with $\alpha p \leq q_{1, j} \leq p$, then the induced subgraph $G\left[U_{1}, U_{2}, U_{3}\right]$ contains at least $(1-\xi) q_{12} q_{13} q_{23}\left|U_{1}\right|\left|U_{2}\right|\left|U_{3}\right|$ copies of $K_{3}$.

Theorem 10 can be deduced from the sparse version of the regularity lemma and Lemma 11 by standard arguments and we omit the details here. Below we deduce Lemma 11 from part $(a)$ of Lemma 9. In the proof we use the following well known consequence of the Cauchy-Schwarz inequality.

Lemma 12. For every $\nu>0$ there exists $\mu>0$ such that for every $m \in \mathbb{N}$ and $x_{1}, \ldots, x_{m}, \bar{x} \in \mathbb{R}$ the following holds. If

$$
\sum_{i \in[m]} x_{i} \geq(1-\mu) \bar{x} m \quad \text { and } \quad \sum_{i \in[m]} x_{i}^{2} \leq(1+\mu) \bar{x}^{2} m,
$$

then

$$
\left|\left\{i \in[m]:\left|x_{i}-\bar{x}\right| \geq \nu \bar{x}\right\}\right| \leq \nu m .
$$

Proof: Lemma $9(a) \Rightarrow$ Lemma 11. Note that the quantifications of the involved constants in Lemma 9 and Lemma 11 are very similar. In fact, there are only two differences. For an application of Lemma 9 we have to make an appropriate choice for $\delta>0$ (depending only on $\alpha$ ) and for the proof of Lemma 11 we are given $\xi>0$.

Assume we are given $\alpha$ and $\xi$ by Lemma 11. First we fix an auxiliary constant

$$
\nu=\frac{\alpha \xi}{3}
$$

and let $\mu=\mu(\nu)$ be given by Lemma 12 . Then we set

$$
\delta=\left(\frac{\alpha^{6} \mu}{1200}\right)^{2}
$$

and let $\varepsilon=\varepsilon(\alpha, \delta)$ be given by Lemma 9 . Following the quantification of Lemma 11 let $\eta$ be given. For this $\eta$ Lemma 9 yields an appropriate choice for $\gamma$. Without loss of generality we may assume that

$$
\gamma \leq \frac{\alpha^{4} \mu \eta^{2}}{144}
$$


Finally, let $n$ be sufficiently large and suppose $p \geq 1 / \sqrt{n}$.

Let $\Gamma, G$, and $U_{i}$ be as in the statement of Lemma 11. We have to show that $G\left[U_{1}, U_{2}, U_{3}\right]$ contains at least $(1-\xi) q_{12} q_{13} q_{23}\left|U_{1}\right|\left|U_{2}\right|\left|U_{3}\right|$ triangles. We will first show that most pairs $\left\{u_{2}, u_{3}\right\} \in E_{\Gamma}\left(U_{2}, U_{3}\right)$ have a "large" joint neighborhood in the graph $G$ in $U_{1}$. For that we study the distribution of the triangles in $\Gamma$ with one edge in $E_{\Gamma}\left(U_{2}, U_{3}\right)$ and the other two edges in $G$. For a pair $\left(u_{2}, u_{3}\right) \in U_{2} \times U_{3}$ we recall that $\operatorname{deg}_{G}\left(u_{2}, u_{3}, U_{1}\right)$ denotes the number of joint neighbors of $u_{2}$ and $u_{3}$ in $U_{1}$ in the graph $G$. Below we will show the following two estimates.

$$
\begin{aligned}
& \sum\left\{\operatorname{deg}_{G}\left(u_{2}, u_{3}, U_{1}\right):\left\{u_{2}, u_{3}\right\} \in E_{\Gamma}\left(U_{2}, U_{3}\right)\right\} \geq\left(1-\frac{\mu}{3}\right) q_{12} q_{13}\left|U_{1}\right| \cdot p\left|U_{2}\right|\left|U_{3}\right|, \\
& \sum\left\{\operatorname{deg}_{G}^{2}\left(u_{2}, u_{3}, U_{1}\right):\left\{u_{2}, u_{3}\right\} \in E_{\Gamma}\left(U_{2}, U_{3}\right)\right\} \leq\left(1+\frac{\mu}{3}\right) q_{12}^{2} q_{13}^{2}\left|U_{1}\right|^{2} \cdot p\left|U_{2}\right|\left|U_{3}\right| .
\end{aligned}
$$

Before we verify (7) and (8), we finish the proof of Lemma 11 based on those estimates.

The $\left(p, \gamma p^{3} n\right)$-jumbledness of $\Gamma$, combined with (6), $\left|U_{2}\right|,\left|U_{3}\right| \geq \eta n$, and $p \leq 1$, yields

$$
\left|e_{\Gamma}\left(U_{2}, U_{3}\right)-p\right| U_{2}|| U_{3}|| \leq \gamma p^{3} n \sqrt{\left|U_{2}\right|\left|U_{3}\right|} \stackrel{(6)}{\leq} \frac{\mu}{3} p^{3}\left|U_{2}\right|\left|U_{3}\right| \leq \frac{\mu}{3} p\left|U_{2}\right|\left|U_{3}\right| .
$$

Note that (7) and (8) show that the assumptions of Lemma 12 are met for $m=$ $e_{\Gamma}\left(U_{2}, U_{3}\right)$ and $\bar{x}=q_{12} q_{13}\left|U_{1}\right|$. Therefore

$$
\operatorname{deg}_{G}\left(u_{2}, u_{3}, U_{1}\right) \geq(1-\nu) q_{12} q_{13}\left|U_{1}\right|
$$

for all but at most $\nu e_{\Gamma}\left(U_{2}, U_{3}\right)$ edges $\left\{u_{2}, u_{3}\right\} \in E_{\Gamma}\left(U_{2}, U_{3}\right)$. Removing those exceptional edges from $G$ yields the following lower bound on the number of triangles with all three edges in $G$ :

$$
\left(e_{G}\left(U_{2}, U_{3}\right)-\nu e_{\Gamma}\left(U_{2}, U_{3}\right)\right) \cdot(1-\nu) q_{12} q_{13}\left|U_{1}\right| .
$$

Since $e_{G}\left(U_{2}, U_{3}\right)=q_{23}\left|U_{2}\right|\left|U_{3}\right| \geq \alpha p\left|U_{2}\right|\left|U_{3}\right|$, the choice of $\nu$ in (4) combined with (9) yields

$$
\left(e_{G}\left(U_{2}, U_{3}\right)-\nu e_{\Gamma}\left(U_{2}, U_{3}\right)\right) \cdot(1-\nu) q_{12} q_{13}\left|U_{1}\right| \geq(1-\xi) q_{12} q_{13} q_{23}\left|U_{1}\right|\left|U_{2}\right|\left|U_{3}\right|,
$$

which concludes the proof of Lemma 11 based on estimates (7) and (8).

Proof of (7). Note that the left-hand side of (7) is the number of triangles in $\Gamma\left[U_{1}, U_{2}, U_{3}\right]$ with both edges incident to a vertex in $U_{1}$ being also present in $G$ and we have

$$
\sum_{\left\{u_{2}, u_{3}\right\} \in E_{\Gamma}\left(U_{2}, U_{3}\right)} \operatorname{deg}_{G}\left(u_{2}, u_{3}, U_{1}\right)=\sum_{u_{1} \in U_{1}} e_{\Gamma}\left(N_{G}\left(u_{1}, U_{2}\right), N_{G}\left(u_{1}, U_{3}\right)\right) .
$$

The $\left(p, \gamma p^{3} n\right)$-jumbledness of $\Gamma$ yields

$$
e_{\Gamma}\left(N_{G}\left(u_{1}, U_{2}\right), N_{G}\left(u_{1}, U_{3}\right)\right) \geq p \operatorname{deg}_{G}\left(u_{1}, U_{2}\right) \operatorname{deg}_{G}\left(u_{1}, U_{3}\right)-\gamma p^{3} n \sqrt{\left|U_{2}\right|\left|U_{3}\right|} .
$$

Moreover, by Lemma $9(a)$, the choice of $\varepsilon$ and the assumption of Lemma 11 imply that $\left(U_{1}, U_{2}\right)_{G}$ and $\left(U_{1}, U_{3}\right)_{G}$ satisfy the pair conditions $\operatorname{PAIR}\left(q_{12}, p, \delta\right)$ and $\operatorname{PAIR}\left(q_{13}, p, \delta\right)$, respectively. In particular, for $j=2$ and 3 ,

$$
\sum_{u_{1} \in U_{1}}\left|\operatorname{deg}_{G}\left(u_{1}, U_{j}\right)-q_{1, j}\right| U_{j}|| \leq \delta p\left|U_{1}\right|\left|U_{j}\right|
$$

As $q_{1, j} \geq \alpha p$ for $j=2$ and 3 , we have

$$
\left|N_{G}\left(u_{1}, U_{j}\right)\right|=\operatorname{deg}_{G}\left(u_{1}, U_{j}\right) \geq(1-\sqrt{\delta}) q_{1, j}\left|U_{j}\right|
$$


for both $j=2$ and $j=3$ for all but at most $(1-2 \sqrt{\delta} / \alpha)\left|U_{1}\right|$ vertices $u_{1} \in U_{1}$. Combining this with (10), (11) and (12) yields

$$
\begin{aligned}
\sum\{ & \left.\operatorname{deg}_{G}\left(u_{2}, u_{3}, U_{1}\right):\left\{u_{2}, u_{3}\right\} \in E_{\Gamma}\left(U_{2}, U_{3}\right)\right\} \\
& \geq(1-2 \sqrt{\delta} / \alpha)\left|U_{1}\right| \cdot p(1-\sqrt{\delta})^{2} q_{12} q_{13}\left|U_{2}\right|\left|U_{3}\right|-\left|U_{1}\right| \gamma p^{3} n \sqrt{\left|U_{2}\right|\left|U_{3}\right|} .
\end{aligned}
$$

The choice of $\delta$ in (5) ensures

$$
(1-2 \sqrt{\delta} / \alpha)(1-\sqrt{\delta})^{2} \geq 1-\mu / 6
$$

and the choice of $\gamma$ in (6) together with $\left|U_{2}\right|,\left|U_{3}\right| \geq \eta n$ and $q_{12}, q_{13} \geq \alpha p$ gives

$$
\left|U_{1}\right| \gamma p^{3} n \sqrt{\left|U_{2}\right|\left|U_{3}\right|} \leq \frac{\mu}{6} p q_{12} q_{13}\left|U_{1}\right|\left|U_{2}\right|\left|U_{3}\right| .
$$

Plugging the last two estimates into (13) yields (7).

In the proof of (8) we will use the following estimate, which is a consequence of the definition of jumbled graphs.

Fact 13. Let $\Gamma=(U \dot{U} V, E)$ be a bipartite $(p, \beta)$-jumbled graph. Let $X \subseteq U$ and $Y \subseteq V$ and $\xi>0$. If $X$ satisfies one of the following two conditions: either $\operatorname{deg}_{\Gamma}(x, Y) \geq(1+\xi) p|Y|$ for all $x \in X$ or $\operatorname{deg}_{\Gamma}(x, Y) \leq(1-\xi) p|Y|$ for all $x \in X$, then

$$
|X| \leq \frac{\beta^{2}}{\xi^{2} p^{2}|Y|}
$$

Proof of (8). We start with rewriting the left-hand side of (8) by summing over all pairs of vertices in $U_{1}$. We obtain

$$
\begin{aligned}
\sum\left\{\operatorname{deg}_{G}^{2}\left(u_{2}, u_{3}, U_{1}\right):\left\{u_{2}, u_{3}\right\}\right. & \left.\in E_{\Gamma}\left(U_{2}, U_{3}\right)\right\} \\
& =\sum_{u_{1} \in U_{1}} \sum_{u_{1}^{\prime} \in U_{1}} e_{\Gamma}\left(\left(N_{G}\left(u_{1}, u_{1}^{\prime}, U_{2}\right), N_{G}\left(u_{1}, u_{1}^{\prime}, U_{3}\right)\right)\right.
\end{aligned}
$$

and, as in the proof of (7), after applying the jumbledness assumption we arrive at

$$
\begin{aligned}
& \sum\left\{\operatorname{deg}_{G}^{2}\left(u_{2}, u_{3}, U_{1}\right):\left\{u_{2}, u_{3}\right\} \in E_{\Gamma}\left(U_{2}, U_{3}\right)\right\} \\
& \quad \leq \sum_{u_{1} \in U_{1}} \sum_{u_{1}^{\prime} \in U_{1}} p D_{G}\left(u_{1}, u_{1}^{\prime}, U_{2}, U_{3}\right)+\gamma p^{3} n \sqrt{D_{G}\left(u_{1}, u_{1}^{\prime}, U_{2}, U_{3}\right)},
\end{aligned}
$$

where we set

$$
D_{G}\left(u_{1}, u_{1}^{\prime}, U_{2}, U_{3}\right)=\operatorname{deg}_{G}\left(u_{1}, u_{1}^{\prime}, U_{2}\right) \operatorname{deg}_{G}\left(u_{1}, u_{1}^{\prime}, U_{3}\right) .
$$

It is left to obtain appropriate estimates on $\operatorname{deg}_{G}\left(u_{1}, u_{1}^{\prime}, U_{2}\right)$ and $\operatorname{deg}_{G}\left(u_{1}, u_{1}^{\prime}, U_{3}\right)$. For that we will appeal to the pair condition, which yields good estimates for those quantities for "most" pairs $\left(u_{1}, u_{1}^{\prime}\right) \in U_{1} \times U_{1}$. For the exceptional pairs $\left(u_{1}, u_{1}^{\prime}\right)$ we will analyze the joint neighborhood of $u_{1}$ and $u_{1}^{\prime}$ in $\Gamma$ and obtain the required bounds from the jumbledness of $\Gamma$. For that we classify the pairs $\left(u_{1}, u_{1}^{\prime}\right)$ according to their degrees in $G$ and $\Gamma$. More precisely, let $\mathcal{G}$ be the "good" pairs having the "right" joint degree in $U_{2}$ and $U_{3}$ :

$$
\mathcal{G}=\left\{\left(u_{1}, u_{1}^{\prime}\right) \in U_{1} \times U_{1}: \operatorname{deg}_{G}\left(u_{1}, u_{1}^{\prime}, U_{j}\right) \leq(1+\sqrt{\delta}) q_{1, j}^{2}\left|U_{j}\right| \text { for } j=2,3\right\} .
$$


Clearly,

$$
\begin{aligned}
& \sum_{\left(u_{1}, u_{1}^{\prime}\right) \in \mathcal{G}} p D_{G}\left(u_{1}, u_{1}^{\prime}, U_{2}, U_{3}\right)+\gamma p^{3} n \sqrt{D_{G}\left(u_{1}, u_{1}^{\prime}, U_{2}, U_{3}\right)} \\
& \leq\left|U_{1}\right|^{2} \cdot p(1+\sqrt{\delta})^{2} q_{12}^{2} q_{13}^{2}\left|U_{2}\right|\left|U_{3}\right|+\left|U_{1}\right|^{2} \cdot \gamma p^{3} n \sqrt{4 q_{12}^{2} q_{13}^{2}\left|U_{2}\right|\left|U_{3}\right|} \\
& \leq\left(1+\frac{\mu}{6}\right) p q_{12}^{2} q_{13}^{2}\left|U_{1}\right|^{2}\left|U_{2}\right|\left|U_{3}\right|,
\end{aligned}
$$

where we used the choice of $\delta$ and $\gamma$ from (5) and (6) and the assumptions on $q_{1, j}$ and $\left|U_{j}\right|$ for $j=2$ and 3 .

Hence, it is left to verify that

$$
\begin{aligned}
\sum_{\left(u_{1}, u_{1}^{\prime}\right) \notin \mathcal{G}} p D_{G}\left(u_{1}, u_{1}^{\prime}, U_{2}, U_{3}\right)+\gamma p^{3} n \sqrt{D_{G}\left(u_{1}, u_{1}^{\prime}, U_{2}, U_{3}\right)} & \\
& \leq \frac{\mu}{6} p q_{12}^{2} q_{13}^{2}\left|U_{1}\right|^{2}\left|U_{2}\right|\left|U_{3}\right| .
\end{aligned}
$$

Lemma 9(a), the choice of $\varepsilon$, and the assumption of Lemma 11 imply that $\left(U_{1}, U_{2}\right)_{G}$ and $\left(U_{1}, U_{3}\right)_{G}$ satisfy $\operatorname{PAIR}\left(q_{12}, p, \delta\right)$ and $\operatorname{PAIR}\left(q_{13}, p, \delta\right)$ respectively. Consequently, we have

$$
|\mathcal{G}| \geq\left(1-2 \sqrt{\delta} / \alpha^{2}\right)\left|U_{1}\right|^{2}
$$

For the contribution of those $2 \sqrt{\delta}\left|U_{1}\right|^{2} / \alpha^{2}$ "exceptional" pairs we analyze the joint neighborhoods of $u_{1}$ and $u_{1}^{\prime}$ in $\Gamma \supseteq G$. Indeed, we will show that (15) stays valid when we replace $G$ by $\Gamma$ in $D_{G}\left(u_{1}, u_{1}^{\prime}, U_{2}, U_{3}\right)$ and show

$$
\begin{aligned}
& \sum_{\left(u_{1}, u_{1}^{\prime}\right) \notin \mathcal{G}} p D_{\Gamma}\left(u_{1}, u_{1}^{\prime}, U_{2}, U_{3}\right)+\gamma p^{3} n \sqrt{D_{\Gamma}\left(u_{1}, u_{1}^{\prime}, U_{2}, U_{3}\right)} \\
& \leq \frac{\mu}{6} p q_{12}^{2} q_{13}^{2}\left|U_{1}\right|^{2}\left|U_{2}\right|\left|U_{3}\right| .
\end{aligned}
$$

We split the pairs in $\left(U_{1} \times U_{1}\right) \backslash \mathcal{G}$ into the following three classes $\mathcal{B}_{1} \dot{\cup} \mathcal{B}_{2} \dot{\cup} \mathcal{B}_{3}$

$$
\begin{aligned}
& \mathcal{B}_{1}=\left\{\left(u_{1}, u_{1}^{\prime}\right) \in\left(U_{1} \times U_{1}\right) \backslash \mathcal{G}: \operatorname{deg}_{\Gamma}\left(u_{1}, u_{1}^{\prime}, U_{j}\right) \leq 4 p^{2}\left|U_{j}\right| \text { for } j=2,3\right\}, \\
& \mathcal{B}_{2}=\left\{\left(u_{1}, u_{1}^{\prime}\right) \in\left(U_{1} \times U_{1}\right) \backslash\left(\mathcal{G} \cup \mathcal{B}_{1}\right): \operatorname{deg}_{\Gamma}\left(u_{1}, U_{j}\right) \leq 2 p\left|U_{j}\right| \text { for } j=2,3\right\},
\end{aligned}
$$

and

$$
\mathcal{B}_{3}=\left(U_{1} \times U_{1}\right) \backslash\left(\mathcal{G} \cup \mathcal{B}_{1} \cup \mathcal{B}_{2}\right) .
$$

Below we bound the contribution to (17) for each class separately.

Contribution of pairs from $\mathcal{B}_{1}$. The definition of $\mathcal{B}_{1}$ and (16) yields

$$
\begin{gathered}
\sum_{\left(u_{1}, u_{1}^{\prime}\right) \in \mathcal{B}_{1}} p D_{\Gamma}\left(u_{1}, u_{1}^{\prime}, U_{2}, U_{3}\right)+\gamma p^{3} n \sqrt{D_{\Gamma}\left(u_{1}, u_{1}^{\prime}, U_{2}, U_{3}\right)} \\
\leq 2 \frac{\sqrt{\delta}}{\alpha^{2}}\left|U_{1}\right|^{2} \cdot\left(16 p^{5}\left|U_{2}\right|\left|U_{3}\right|+\gamma p^{3} n \sqrt{16 p^{4}\left|U_{2}\right|\left|U_{3}\right|}\right) \\
\leq \frac{\mu}{18} p q_{12}^{2} q_{13}^{2}\left|U_{1}\right|^{2}\left|U_{2}\right|\left|U_{3}\right|,
\end{gathered}
$$

because of the choice of $\delta$ in (5) and $\gamma \leq \eta / 36$ in (6) and the assumptions on $q_{1 j}$ and $\left|U_{j}\right|$ for $j=2$ and 3 . 
Contribution of pairs from $\mathcal{B}_{2}$. Fix $j \in\{2,3\}$ and let $u_{1} \in U_{1}$ be a vertex with $\operatorname{deg}_{\Gamma}\left(u_{1}, U_{j}\right) \leq 2 p\left|U_{j}\right|$. Let $X\left(u_{1}, j\right)$ be the set of vertices $u_{1}^{\prime} \in U_{1}$ with

$$
\operatorname{deg}_{\Gamma}\left(u_{1}, u_{1}^{\prime}, U_{j}\right)>4 p^{2}\left|U_{j}\right| .
$$

As $\Gamma$ is $\left(p, \gamma p^{3} n\right)$-jumbled, Fact 13 applied to $X\left(u_{1}, j\right)$ and a superset $Y\left(u_{1}, j\right) \supseteq$ $N_{\Gamma}\left(u_{1}, U_{j}\right)$ in $U_{j}$ containing precisely $2 p\left|U_{j}\right|$ vertices gives

$$
\left|X\left(u_{1}, j\right)\right| \leq \frac{\gamma^{2} p^{6} n^{2}}{p^{2} \cdot 2 p\left|U_{j}\right|}=\frac{\gamma^{2} p^{3} n^{2}}{2\left|U_{j}\right|} .
$$

Consequently, since $\gamma \leq \eta^{2}$ and $\left|U_{j}\right| \geq \eta n$ for $j=1,2,3$ we have

$$
\left|\mathcal{B}_{2}\right| \leq\left|U_{1}\right|\left(\frac{\gamma^{2} p^{3} n^{2}}{2\left|U_{2}\right|}+\frac{\gamma^{2} p^{3} n^{2}}{2\left|U_{3}\right|}\right) \leq \gamma p^{3}\left|U_{1}\right|^{2}
$$

Finally, since $\operatorname{deg}_{\Gamma}\left(u_{1}, u_{1}^{\prime}, U_{j}\right) \leq \operatorname{deg}_{\Gamma}\left(u_{1}, U_{j}\right) \leq 2 p\left|U_{j}\right|$ for all $\left(u_{1}, u_{1}^{\prime}\right) \in \mathcal{B}_{2}$ and $j=2,3$ we have

$$
\begin{gathered}
\sum_{\left(u_{1}, u_{1}^{\prime}\right) \in \mathcal{B}_{2}} p D_{\Gamma}\left(u_{1}, u_{1}^{\prime}, U_{2}, U_{3}\right)+\gamma p^{3} n \sqrt{D_{\Gamma}\left(u_{1}, u_{1}^{\prime}, U_{2}, U_{3}\right)} \\
\leq\left|\mathcal{B}_{2}\right| \cdot\left(4 p^{3}\left|U_{2}\right|\left|U_{3}\right|+\gamma p^{3} n \sqrt{4 p^{2}\left|U_{2}\right|\left|U_{3}\right|}\right) \\
\leq \frac{\mu}{18} p q_{12}^{2} q_{13}^{2}\left|U_{1}\right|^{2}\left|U_{2}\right|\left|U_{3}\right|,
\end{gathered}
$$

where we use the choice of $\gamma$ from (5) and the assumptions on $q_{1, j}$ and $\left|U_{j}\right|$ for $j=2$ and 3 .

Contribution of pairs from $\mathcal{B}_{3}$. The analysis for those pairs is similar to the one in the last case. For $j \in\{2,3\}$ let $X_{j}$ be the vertices $u_{1} \in U_{1}$ with

$$
\operatorname{deg}_{\Gamma}\left(u_{1}, U_{j}\right)>2 p\left|U_{j}\right| .
$$

Fact 13 applied to $X_{j}$ and $Y=U_{j}$ yields

$$
\left|X_{j}\right| \leq \frac{\gamma^{2} p^{6} n^{2}}{p^{2}\left|U_{j}\right|} \leq \gamma p^{4}\left|U_{1}\right|,
$$

since $\gamma \leq \eta^{2}$. Therefore, we have $\left|\mathcal{B}_{3}\right| \leq 2 \gamma p^{4}\left|U_{1}\right|^{2}$ and since $\operatorname{deg}_{\Gamma}\left(u_{1}, u_{1}^{\prime}, U_{j}\right) \leq\left|U_{j}\right|$ for $j=2$ and 3 we have

$$
\begin{gathered}
\sum_{\left(u_{1}, u_{1}^{\prime}\right) \in \mathcal{B}_{2}} p D_{\Gamma}\left(u_{1}, u_{1}^{\prime}, U_{2}, U_{3}\right)+\gamma p^{3} n \sqrt{D_{\Gamma}\left(u_{1}, u_{1}^{\prime}, U_{2}, U_{3}\right)} \\
\leq 2 \gamma p^{4}\left|U_{1}\right|^{2} \cdot\left(p\left|U_{2}\right|\left|U_{3}\right|+\gamma p^{3} n \sqrt{\left|U_{2}\right|\left|U_{3}\right|}\right) \\
\leq \frac{\mu}{18} p q_{12}^{2} q_{13}^{2}\left|U_{1}\right|^{2}\left|U_{2}\right|\left|U_{3}\right|,
\end{gathered}
$$

where we use $\gamma \leq \alpha^{4} \mu \eta / 72$.

Finally, we note that (18), (19) and (20) implies (17), which concludes the proof of (8). 


\section{Roth'S THEOREM FOR PSEUDORANDOM SETS OF INTEGERS}

We prove Theorem 4 in this section. We shall in fact consider pseudorandom subsets of finite abelian groups. Throughout this section $\mathbb{G}$ is a finite abelian group, of order $n=|\mathbb{G}|$, which is assumed to be odd. (Roth's theorem was generalized to this setting by Brown and Buhler [4]. For a proof based on the triangle removal lemma, see [11] and for better quantitative bounds see [20, 19].)

Let $\chi_{0}, \ldots, \chi_{n-1}: \mathbb{G} \rightarrow \mathbb{C}$ be the $n$ characters of $\mathbb{G}$. We suppose $\chi_{0}$ is the principal character, that is, $\chi_{0}(g)=1$ for all $g \in \mathbb{G}$.

Suppose now that we are given a set $A \subseteq \mathbb{G}$. Let

$$
\lambda_{k}(A)=\sum_{a \in A} \chi_{k}(a) \in \mathbb{C}
$$

for all $0 \leq k<n$. Clearly, $\lambda_{0}(A)=|A|$. If $\left|\lambda_{k}(A)\right| \leq \lambda$ for all $1 \leq k<n$, we say that $A$ is an $(n, \lambda)$-subset of $\mathbb{G}$ or an $(n, \lambda)$-set for short. We let

$$
r_{3}(A)=\max \left\{|B|: B \text { is an } \mathrm{AP}_{3} \text {-free subset of } A\right\} .
$$

We shall prove the following result.

Theorem 14. For every $\delta>0$ there exist $\gamma>0$ and $n_{0}$ such that for every odd $n \geq n_{0}$ the following holds.

Suppose $A$ is an $(n, \lambda)$-subset of an abelian group $\mathbb{G}$ of order $n$ and

$$
\lambda \leq \gamma \frac{|A|^{3}}{n^{2}}
$$

Then $r_{3}(A) \leq \delta|A|$.

The proof of Theorem 14 will be given in Section 4.2 .

4.1. Proof of Theorem 4. Let us deduce Theorem 4 from Theorem 14. Let us first specialize the latter theorem to $\mathbb{G}=\mathbb{Z} / n \mathbb{Z}$. Let $\vartheta=\exp (2 \pi \mathrm{i} / n)$. The characters $\chi_{k}(0 \leq k<n)$ of $\mathbb{Z} / n \mathbb{Z}$ are given by

$$
\chi_{k}(x)=\vartheta^{k x} \quad(x \in \mathbb{Z} / n \mathbb{Z}) .
$$

Therefore, the $\lambda_{k}(A)$ in (21) are given by (1). We conclude that the hypothesis in Theorem 4 simply says that $A$ is an $(n, \lambda)$-subset of $\mathbb{Z} / n \mathbb{Z}$ satisfying (23). Theorem 14 tells us that, for any $\delta>0$, for suitable $\gamma$ and $n_{0}$ we have $r_{3}(A) \leq \delta|A|$.

To finish the proof of Theorem 4 , we have to deal with the issue that an arithmetic progression in $\mathbb{Z} / n \mathbb{Z}$ is not necessarily an arithmetic progression in the integers. For instance, $(5,2,6)$ is a 3 -term arithmetic progression with difference 4 modulo 7 , as is $(1,5,2)$. This is easy to handle, by making use of the following easy fact: let $(a, b, c)$ be a triple of distinct integers with $0 \leq a, b, c \leq n / 2$ or with $n / 2<a, b, c \leq n$, and suppose that the residue classes of $a, b$ and $c$ modulo $n$ form a 3-term arithmetic progression, i.e., $a+c \equiv 2 b(\bmod n)$. Then $(a, b, c)$ is an arithmetic progression in $[n]$, i.e., $a+c=2 b$.

Since, for any $B \subseteq[n]$, either $|B \cap\{1, \ldots,\lfloor n / 2\rfloor\}| \geq|B| / 2$ or else we have $|B \cap\{\lceil n / 2\rceil, \ldots, n\}| \geq|B| / 2$, Theorem 14 applied to subsets of $\mathbb{Z} / n \mathbb{Z}$, implies the desired result for subsets on $[n]$. 
4.2. Proof of Theorem 14. We shall present the proof of Theorem 14 in this section.

We shall make use of a certain tripartite graph $\Gamma=\Gamma(A)$, defined in terms of the given $(n, \lambda)$-set $A \subseteq \mathbb{G}$.

Definition 15 (Graph $\Gamma=\Gamma(A)$ ). The tripartite graph $\Gamma=\Gamma(A)$ has vertex classes $X_{1}, X_{2}$, and $X_{3}$, where each $X_{i}$ is a disjoint copy of $\mathbb{G}$. For every $x_{1} \in X_{1}$, we join $x_{1}$ to the vertices $x_{1}+a \in X_{2}$ and $x_{1}+2 a \in X_{3}$ for all $a \in A$. Moreover, we join every $x_{2} \in X_{2}$ to the vertex $x_{2}+a \in X_{3}$ for all $a \in A$.

It is clear that the bipartite graph $\Gamma\left[X_{1} \cup X_{2}\right]$ induced by $X_{1} \cup X_{2}$ in $\Gamma$ and the bipartite graph $\Gamma\left[X_{2} \cup X_{3}\right]$ induced by $X_{2} \cup X_{3}$ in $\Gamma$ are isomorphic in a natural way. The bipartite graph $\Gamma\left[X_{1} \cup X_{3}\right]$ induced by $X_{1} \cup X_{3}$ in $\Gamma$ is "similar" to those bipartite graphs, but one uses, so to speak, the set $2 A$ instead of $A$ to obtain this graph. In what follows, we study these bipartite graphs $\Gamma_{1}(A) \simeq \Gamma\left[X_{1} \cup X_{2}\right] \simeq \Gamma\left[X_{2} \cup X_{3}\right]$ and $\Gamma_{2}(2 A) \simeq \Gamma\left[X_{1} \cup X_{3}\right]$ that form $\Gamma$.

4.2.1. The graph $\Gamma_{1}=\Gamma_{1}(A)$. The two vertex classes of $\Gamma_{1}=\Gamma_{1}(A)$ are two disjoint copies $X$ and $Y$ of $\mathbb{G}$. A vertex $x \in X$ is joined to a vertex $y \in Y$ if $y-x \in A$. Thus, the edges of $\Gamma_{1}=\Gamma_{1}(A)=\left(X \dot{\cup} Y, E_{1}\right)$ are of the form $(x, x+a) \in X \times Y$, where $x \in X$ and $a \in A$. It is clear that $\Gamma_{1}$ is $|A|$-regular. The graph $\Gamma=\Gamma(A)$ defined above contains two copies of $\Gamma_{1}(A)$.

A key fact about $\Gamma_{1}=\Gamma_{1}(A)$ is given in the following lemma, which is a bipartite version of the so called expander mixing lemma (see, e.g., [2, Corollary 9.2.5]).

Lemma 16. Suppose $A$ is an $(n, \lambda)$-subset of $\mathbb{G}$ and let $\Gamma_{1}=\Gamma_{1}(A)=\left(X \dot{\cup} Y, E_{1}\right)$ be as defined above. Then, for any $X^{\prime} \subseteq X$ and any $Y^{\prime} \subseteq Y$, we have

$$
\left|e\left(X^{\prime}, Y^{\prime}\right)-\frac{|A|}{n}\right| X^{\prime}|| Y^{\prime}|| \leq \lambda \sqrt{\left|X^{\prime}\right|\left|Y^{\prime}\right|} .
$$

The proof of Lemma 16 is given in Section 4.2.4.

4.2.2. The graph $\Gamma_{2}=\Gamma_{2}(2 A)$. Consider $2 A=\{2 a: a \in A\}$ and let $\Gamma_{2}=\Gamma_{2}(2 A)$ be defined as follows: the vertex classes of $\Gamma_{2}$ are two disjoint copies $X$ and $Y$ of $\mathbb{G}$, and a vertex $x \in X$ is joined to a vertex $y \in Y$ if $y-x \in 2 A$. Thus, the edges of $\Gamma_{2}=\Gamma_{2}(2 A)=\left(X \dot{\cup} Y, E_{2}\right)$ are of the form $(x, x+2 a) \in X \times Y$, where $x \in X$ and $a \in A$. Since $n$ is odd, the map $g \mapsto 2 g$ is a bijection on $\mathbb{G}$, and hence $|2 A|=|A|$. Therefore, $\Gamma_{2}=\Gamma_{2}(2 A)$ is $|A|$-regular. The graph $\Gamma=\Gamma(A)$ defined above contains a copy of $\Gamma_{2}=\Gamma_{2}(2 A)$.

Observe now that, for all $k$, we have

$$
\lambda_{k}(2 A)=\sum_{a \in A} \chi_{k}(2 a)=\sum_{a \in A} \chi_{k}(a)^{2} .
$$

Recall that the characters $\chi_{k}(0 \leq k<n)$ form a group $\hat{\mathbb{G}}$ under pointwise multiplication. As $|\hat{\mathbb{G}}|=n$ is odd, the map $\chi_{k} \mapsto \chi_{k}^{2}$ is a bijection on $\hat{\mathbb{G}}$, fixing the principal character $\chi_{0}$. Therefore,

$$
\left\{\lambda_{k}(A): 1 \leq k<n\right\}=\left\{\lambda_{k}(2 A): 1 \leq k<n\right\} .
$$

Moreover, $\lambda_{0}(2 A)=|2 A|=|A|$. We have just proved the following fact.

Lemma 17. If $A \subseteq \mathbb{G}$ is an $(n, \lambda)$-set, then so is $2 A$ and, in particular, inequality (25) holds for $\Gamma_{2}=\Gamma_{2}(2 A)=\left(X \dot{\cup} Y, E_{2}\right)$ for any $X^{\prime} \subseteq X$ and any $Y^{\prime} \subseteq Y$. 
An immediate corollary of Lemmas 16 and 17 is the following crucial fact about the graph $\Gamma=\Gamma(A)$ (see Definition 15).

Corollary 18. Let $A \subseteq \mathbb{G}$ be an $(n, \lambda)$-set. Then $\Gamma=\Gamma(A)$ is a $(|A| / n, \lambda)$-jumbled tripartite graph (see Definition 8).

4.2.3. Proof of Theorem 14. To prove Theorem 14, it suffices to run the well known derivation of Roth's theorem from the triangle removal lemma (see, e.g., [25]) in our context. Theorem 10 and Corollary 18 will be crucial here.

For convenience, let us first make a simple remark, whose proof is included for completeness.

Fact 19. Let $A \subseteq \mathbb{G}$ be an $(n, \lambda)$-set, with $|A| \leq 3 n / 4$. Then $\lambda \geq \sqrt{|A|} / 2$.

Proof. We follow a proof in, e.g., [18]. Let $M=\left(m_{x y}\right) \in\{0,1\}^{n \times n}$ be a $\{0,1\}$ matrix with rows and columns indexed by the group elements of $\mathbb{G}$ and

$$
m_{x y}= \begin{cases}1, & \text { if } y-x \in A \\ 0, & \text { otherwise }\end{cases}
$$

We denote by $M^{*}$ the conjugate transpose of $M$. Computing the $\operatorname{trace} \operatorname{tr}\left(M^{*} M\right)$ of $M^{*} M$ in two ways, we obtain that

$$
n|A|=\operatorname{tr}\left(M^{*} M\right)=\sum_{0 \leq k<n} \lambda_{k}^{2}=|A|^{2}+\sum_{1 \leq k<n} \lambda_{k}^{2} \leq|A|^{2}+\lambda^{2}(n-1),
$$

whence $\lambda^{2} \geq|A|(1-|A| / n) \geq|A| / 4$, and the result follows.

Proof of Theorem 14. Let $\delta>0$ be given. We invoke Theorem 10 with $\delta / 3$ and obtain positive constants $c$ and $\gamma$ and let $n$ be a sufficiently large odd integer.

Let $A \subseteq \mathbb{G}$ be an $(n, \lambda)$-set satisfying (23). Without loss of generality we may assume that $|A| \leq 3 n / 4$, since, for any $\xi>0$ and $|A| \geq \xi n$, Theorem 14 follows from the result of Brown and Buhler [4].

Therefore Fact 19 implies that the $(n, \lambda)$-sets $A$ must satisfy $\lambda \geq \sqrt{|A|} / 2$. Consequently, (23) implies that $\gamma|A|^{3} / n^{2} \geq \sqrt{|A|} / 2$, which gives

$$
|A| \geq(2 \gamma)^{-2 / 5} n^{4 / 5}>\sqrt{n} .
$$

Let $p=|A| / n$. We have just seen that $p>1 / \sqrt{n}$.

Suppose $B \subseteq A$ is such that $|B|>\delta|A|$. We shall show that $B$ contains a 3 -term arithmetic progression.

In order to do so, we consider the graphs $\Gamma=\Gamma(A)$ and $G=\Gamma(B)$. I.e., $G$ is a tripartite graph with the vertex set $X_{1} \dot{\cup} X_{2} \dot{\cup} X_{3}$ consisting of three disjoint copies of $\mathbb{G}$ and $\{x, y\}$ is an edge in $G\left[X_{1} \cup \dot{\cup} X_{2}\right]$ and $G\left[X_{2} \dot{\cup} X_{3}\right]$ if $y-x \in B$ and it is an edge in $G\left[X_{1} \cup \dot{\cup} X_{3}\right]$ if $y-x$ in $2 B$. Clearly, $G$ is a subgraph of $\Gamma=\Gamma(A)$.

Corollary 18 tells us that $\Gamma=\Gamma(A)$ is a $(p, \lambda)$-jumbled tripartite graph. Together with assumption (23), this implies that $\Gamma$ is a $\left(p, \gamma p^{3} n\right)$-jumbled tripartite graph. In view of Theorem 10, this implies that $G \subseteq \Gamma$ satisfies one of the following properties:

(*) either $G$ contains more than $c p^{3} n^{3}=c|A|^{3}$ triangles, or else

$$
\tau_{3}(G) \leq \frac{\delta}{3} e(\Gamma)=\delta n|A|
$$


(recall that we defined $c$ and $\gamma$ invoking Theorem 10 with $\delta / 3$ ).

Clearly, $G$ is a spanning subgraph of $\Gamma$ and the edges of $G$ are of the form $\left(x_{1}, x_{1}+b\right) \in X_{1} \times X_{2},\left(x_{1}, x_{1}+2 b\right) \in X_{1} \times X_{3}$, and $\left(x_{2}, x_{2}+b\right) \in X_{2} \times X_{3}$, where $x_{1} \in X_{1}, x_{2} \in X_{2}$, and $b \in B$.

The graph $G$ contains some triangles that are trivial: triangles whose vertex sets are of the form $\left\{x_{1}, x_{1}+b, x_{1}+2 b\right\}$, where $x_{1} \in X_{1}, x_{1}+b \in X_{2}$, and $x_{1}+2 b \in$ $X_{3}$. Clearly, these triangles are pairwise edge-disjoint. Since there are $n|B|$ such triangles, we see that $\tau_{3}(G) \geq n|B|>\delta n|A|$. In view of assertion (*) above, we deduce that $G$ contains more than $c p^{3} n^{3}=c|A|^{3}$ triangles. Now note that, because of (29), we have $c|A|^{3} \gg n|A| \geq n|B|$, that is, the total number of triangles in $G$ is larger than the number of trivial triangles in $G$. Therefore $G$ contains a nontrivial triangle. Let the vertex set of such a non-trivial triangle be $\left\{x_{1}, x_{2}, x_{3}\right\}$ (with $x_{i} \in X_{i}$ for all $i$ ). Then, for some $b_{1}, b_{2}$, and $b_{3} \in B$ with $b_{1} \neq b_{3}$, we have $x_{2}=x_{1}+b_{1}, x_{3}=x_{1}+2 b_{2}=x_{2}+b_{3}$. We deduce that $b_{1}+b_{3}=2 b_{2}$, and hence $\left(b_{1}, b_{2}, b_{3}\right)$ is a 3 -term arithmetic progression in $B$, as required.

4.2.4. Proof of Lemma 16. We follow the proof of the expander mixing lemma in, e.g., [18] (see also [2]). We state and prove an auxiliary lemma before turning to Lemma 16.

Let $M=\left(m_{x y}\right)_{x \in X, y \in Y}$ be the $\{0,1\}$-bipartite adjacency matrix of $\Gamma_{1}=\Gamma_{1}(A)$, by which we mean that $m_{x y}=1$ if $x$ and $y$ are adjacent in $\Gamma_{1}$ and $m_{x y}=0$ otherwise. Hence $m_{x y}=\mathbb{1}_{A}(y-x)$. Let us consider the characters $\chi_{k}$ as column vectors, with the $x$ th entry equal to $\chi_{k}(x)$. Let us also set

$$
\mathbf{u}_{k}=\frac{1}{\sqrt{n}} \chi_{k} \in \mathbb{C}^{n}
$$

for all $0 \leq k<n$. Moreover, let $U$ be $n \times n$ matrix whose $k$ th column is $\mathbf{u}_{k}$. In what follows, we use the standard notation $U^{*}$ to denote the conjugate transpose of $U$.

Lemma 20. Let $M, \mathbf{u}_{0}, \ldots, \mathbf{u}_{n-1}$, and $U$ be as above. Then the following holds:

(i) The eigenvalues of $M$ are the $\lambda_{k}(A)(0 \leq k<n)$, with associated eigenvectors $\mathbf{u}_{k}(0 \leq k<n)$.

(ii) The $\mathbf{u}_{k}(0 \leq k<n)$ form an orthonormal basis of $\mathbb{C}^{n}$. Thus $U^{*} U=I_{n}$, where $I_{n}$ is the $n \times n$ identity matrix.

(iii) We have

$$
M=\sum_{0 \leq k<n} \lambda_{k} \mathbf{u}_{k} \mathbf{u}_{k}^{*}
$$

Proof. Let us compute the product $M \chi_{k}$. For any $x \in \mathbb{G}$, we have

$$
\begin{aligned}
\left(M \chi_{k}\right)_{x}=\sum_{y \in \mathbb{G}} m_{x y} \chi_{k}(y)=\sum_{y \in \mathbb{G}} \mathbb{1}(y-x) \chi_{k}(y)=\sum_{a \in A} \chi_{k}(x+a) & \\
& =\chi_{k}(x) \sum_{a \in A} \chi_{k}(a)=\chi_{k}(x) \lambda_{k}(A),
\end{aligned}
$$

whence $(i)$ follows. For $(i i)$, we recall that, for any $k$ and $\ell$, we have

$$
\sum_{x \in \mathbb{G}} \chi_{k}(x) \overline{\chi_{\ell}(x)}= \begin{cases}n & \text { if } k=\ell \\ 0 & \text { otherwise. }\end{cases}
$$


Therefore ( $i i)$ follows. Finally, let us consider $(i i i)$. Since the $\mathbf{u}_{k}$ form a basis of $\mathbb{C}^{n}$, it suffices to check that $M$ and the matrix on the right-hand side of (31) act the same way on the $\mathbf{u}_{k}$. For any fixed $0 \leq \ell<n$, recalling $(i i)$, we see that

$$
\left(\sum_{0 \leq k<n} \lambda_{k} \mathbf{u}_{k} \mathbf{u}_{k}^{*}\right) \mathbf{u}_{\ell}=\lambda_{\ell} \mathbf{u}_{\ell} \mathbf{u}_{\ell}^{*} \mathbf{u}_{\ell}=\lambda_{\ell} \mathbf{u}_{\ell}=M \mathbf{u}_{\ell}
$$

Identity (31) follows and ( $i i i)$ is proved.

We are now ready to prove Lemma 16.

Proof of Lemma 16. Using part (iii) of Lemma 20, we can write $M$ as $M_{0}+E$, where $M_{0}=\lambda_{0} \mathbf{u}_{0} \mathbf{u}_{0}^{*}$ and $E=\sum_{0<k<n} \lambda_{k} \mathbf{u}_{k} \mathbf{u}_{k}^{*}$. Note that, then,

$$
M_{0}=\lambda_{0} \mathbf{u}_{0} \mathbf{u}_{0}^{*}=|A| \chi_{0} \chi_{0}^{*} / n=(|A| / n) J_{n},
$$

where $J_{n}$ is the $n \times n$ matrix all whose entries are 1 .

To prove (25), we let $X^{\prime} \subseteq X$ and $Y^{\prime} \subseteq Y$ be fixed. It is clear that

$$
e\left(X^{\prime}, Y^{\prime}\right)=\mathbb{1}_{X^{\prime}}^{*} M \mathbb{1}_{Y^{\prime}}=\mathbb{1}_{X^{\prime}}^{*} M_{0} \mathbb{1}_{Y^{\prime}}+\mathbb{1}_{X^{\prime}}^{*} E \mathbb{1}_{Y^{\prime}} .
$$

In view of (35), we have

$$
\mathbb{1}_{X^{\prime}}^{*} M_{0} \mathbb{1}_{Y^{\prime}}=\frac{|A|}{n} \mathbb{1}_{X^{\prime}}^{*} J_{n} \mathbb{1}_{Y^{\prime}}=\frac{|A|}{n}\left|X^{\prime}\right|\left|Y^{\prime}\right| .
$$

Now let us write $\mathbb{1}_{X^{\prime}}$ and $\mathbb{1}_{Y^{\prime}}$ in the orthonormal basis formed by the $\mathbf{u}_{k}(0 \leq k<n)$ :

$$
\mathbb{1}_{X^{\prime}}=\sum_{0 \leq k<n} \alpha_{k} \mathbf{u}_{k}
$$

and

$$
\mathbb{1}_{Y^{\prime}}=\sum_{0 \leq k<n} \beta_{k} \mathbf{u}_{k}
$$

Again, by the orthonormality of the $\mathbf{u}_{k}$, we have

$$
\begin{array}{r}
\left|\mathbb{1}_{X^{\prime}}^{*} E \mathbb{1}_{Y^{\prime}}\right|=\left|\left(\sum_{0 \leq j<n} \alpha_{j} \mathbf{u}_{j}\right)\left(\sum_{0<k<n} \lambda_{k} \mathbf{u}_{k} \mathbf{u}_{k}^{*}\right)\left(\sum_{0 \leq \ell<n} \beta_{\ell} \mathbf{u}_{\ell}\right)\right| \\
=\left|\sum_{1 \leq k<n} \lambda_{k} \alpha_{k} \beta_{k}\right| \leq \sum_{1 \leq k<n}\left|\lambda_{k} \alpha_{k} \beta_{k}\right| \leq \lambda \sum_{1 \leq k<n}\left|\alpha_{k} \beta_{k}\right|
\end{array}
$$

which, by the Cauchy-Schwarz inequality, is at most

$$
\lambda \sqrt{\sum_{1 \leq k<n}\left|\alpha_{k}\right|^{2}} \sqrt{\sum_{1 \leq k<n}\left|\beta_{k}\right|^{2}} \leq \lambda\left\|\mathbb{1}_{X^{\prime}}\right\|_{2}\left\|\mathbb{1}_{Y^{\prime}}\right\|_{2}=\lambda \sqrt{\left|X^{\prime}\right|} \sqrt{\left|Y^{\prime}\right|}
$$

Inequality (25) follows from (36), (37), (40), and (41).

\section{Proof of the key technical lemma}

Section 5.1 is devoted to the proof of part $(b)$ of Lemma 9. The main tool in the proof of part $(a)$ of Lemma 9 is Lemma 21. We discuss Lemma 21 in Section 5.2 and deduce Lemma $9(a)$ from Lemma 21 in Section 5.3. The proof of Lemma 21 is deferred to Section 5.4. 
5.1. PAIR implies DISC. In this section we prove implication $(b)$ of Lemma 9. The proof of this implication "imitates" the well known proof of the dense case, i.e., of the implication $(b)$ of Theorem 7.

Proof of Lemma 9 part $(b)$. Let $\alpha$ and $\delta>0$ be given. Applying Lemma 12 with $\nu=\delta / 4$ we obtain $\mu>0$ and we set

$$
\varepsilon=\alpha^{2} \delta^{2} \mu / 4
$$

For any given $\eta>0$, we let

$$
\gamma=\delta \eta / 2
$$

Finally, let $n$ be sufficiently large and $p \geq 1 / \sqrt{n}$.

Let $\Gamma=\left(U \dot{U} V, E_{\Gamma}\right), G=\left(U \dot{\cup} V, E_{\Gamma}\right)$, and sets $X \subseteq U$ and $Y \subseteq V$ satisfy assumptions $(i)-(i i i)$ of Lemma 9. Moreover, we suppose $\Gamma$ is $\left(p, \gamma p^{3 / 2} n\right)$-jumbled and $(X, Y)_{G}$ satisfies $\operatorname{PAIR}(q, p, \varepsilon)$ for some $q \in[\alpha p, p]$, i.e.,

$$
\sum_{x \in X}\left|\operatorname{deg}_{G}(x, Y)-q\right| Y|| \leq \varepsilon p|X||Y|
$$

and

$$
\sum_{x \in X} \sum_{x^{\prime} \in X}\left|\operatorname{deg}_{G}\left(x, x^{\prime}, Y\right)-q^{2}\right| Y|| \leq \varepsilon p^{2}|X|^{2}|Y|
$$

Let $X^{\prime} \subseteq X$ and $Y^{\prime} \subseteq Y$. We will show that

$$
\left|e_{G}\left(X^{\prime}, Y^{\prime}\right)-q\right| X^{\prime}|| Y^{\prime}|| \leq \delta p|X||Y| .
$$

First we consider the case in which at least one of the sets $X^{\prime}$ or $Y^{\prime}$ is small. Suppose $\left|X^{\prime}\right|<\delta|X| / 2$ or $\left|Y^{\prime}\right|<\delta|Y| / 2$. In this case we have

$$
q\left|X^{\prime}\right|\left|Y^{\prime}\right| \leq \delta q|X||Y| \leq \delta p|X||Y| .
$$

Moreover, we infer from the $\left(p, \gamma p^{3 / 2} n\right)$-jumbledness of $\Gamma$ combined with $|X|,|Y| \geq$ $\eta n$, and $p \leq 1$ that

$$
\begin{aligned}
e_{G}\left(X^{\prime}, Y^{\prime}\right) & \leq e_{\Gamma}\left(X^{\prime}, Y^{\prime}\right) \leq p\left|X^{\prime}\right|\left|Y^{\prime}\right|+\gamma p^{3 / 2} n \sqrt{\left|X^{\prime}\right|\left|Y^{\prime}\right|} \\
& \leq \frac{\delta}{2} p|X||Y|+\frac{\gamma}{\eta} p|X||Y| \stackrel{(43)}{\leq} \delta p|X||Y| \leq q\left|X^{\prime}\right|\left|Y^{\prime}\right|+\delta p|X||Y|,
\end{aligned}
$$

which yields (46) for this case.

Now we assume $\left|X^{\prime}\right| \geq \delta|X| / 2$ and $\left|Y^{\prime}\right| \geq \delta|Y| / 2$. In this case (44) yields

$$
\sum_{x \in X^{\prime}}\left|\operatorname{deg}_{G}(x, Y)-q\right| Y|| \leq \sum_{x \in X}\left|\operatorname{deg}_{G}(x, Y)-q\right| Y|| \leq \varepsilon p|X||Y| \stackrel{(42)}{\leq} \mu q\left|X^{\prime}\right||Y|
$$

and, therefore,

$$
\sum_{y \in Y} \operatorname{deg}_{G}\left(y, X^{\prime}\right)=\sum_{x \in X^{\prime}} \operatorname{deg}_{G}(x, Y) \geq(1-\mu) q\left|X^{\prime}\right||Y| .
$$

Similarly, since $X^{\prime} \subseteq X$, (45) yields

$$
\sum_{x \in X^{\prime}} \sum_{x^{\prime} \in X^{\prime}}\left|\operatorname{deg}_{G}\left(x, x^{\prime}, Y\right)-q^{2}\right| Y|| \leq \varepsilon p^{2}|X|^{2}|Y| \stackrel{(42)}{\leq} \mu q^{2}\left|X^{\prime}\right|^{2}|Y|
$$

and, therefore,

$$
\sum_{y \in Y} \operatorname{deg}_{G}^{2}\left(y, X^{\prime}\right)=\sum_{x \in X^{\prime}} \sum_{x^{\prime} \in X^{\prime}} \operatorname{deg}_{G}\left(x, x^{\prime}, Y\right) \leq(1+\mu) q^{2}\left|X^{\prime}\right|^{2}|Y| .
$$


Owing to (47) and (48), we infer from Lemma 12 that all but at most $\nu|Y|$ vertices $y \in Y$ satisfy

$$
\left|\operatorname{deg}_{G}\left(y, X^{\prime}\right)-q\right| X^{\prime}|| \leq \nu q\left|X^{\prime}\right| .
$$

Since we chose $\nu<\delta / 2$, we have

$$
e_{G}\left(X^{\prime}, Y^{\prime}\right) \geq(1-\nu)\left|Y^{\prime}\right| \cdot(1-\nu) q\left|X^{\prime}\right| \geq(1-\delta) q\left|X^{\prime}\right|\left|Y^{\prime}\right| \geq q\left|X^{\prime}\right| Y^{\prime}|-\delta p| X|| Y \mid .
$$

On the other hand, the $\left(p, \gamma p^{3 / 2} n\right)$-jumbledness of $\Gamma$ yields by Fact 13 that all but at most

$$
\frac{\gamma^{2} p^{3} n^{2}}{p^{2}\left|X^{\prime}\right|} \leq \frac{\gamma^{2} p n^{2}}{\delta|X|} \leq \frac{\gamma^{2} p n^{2}}{\delta \eta n} \leq \frac{\gamma^{2} p|Y|}{\delta \eta^{2}} \stackrel{(43)}{\leq} \frac{\delta}{4} p|Y|
$$

vertices $y \in Y^{\prime}$ we have

$$
\operatorname{deg}_{G}\left(y, X^{\prime}\right) \leq \operatorname{deg}_{\Gamma}\left(y, X^{\prime}\right) \leq 2 p\left|X^{\prime}\right| .
$$

Consequently, owing to (49), (51), and (52) we have

$$
\begin{aligned}
e_{G}\left(X^{\prime}, Y^{\prime}\right) & \leq\left|Y^{\prime}\right| \cdot(1+\nu) q\left|X^{\prime}\right|+\nu\left|Y^{\prime}\right| \cdot 2 p\left|X^{\prime}\right|+\frac{\delta}{4} p|Y| \cdot\left|X^{\prime}\right| \\
& \leq q\left|X^{\prime}\right|\left|Y^{\prime}\right|+3 \nu p|X||Y|+\frac{\delta}{4} p|Y||X| \leq q\left|X^{\prime}\right|\left|Y^{\prime}\right|+\delta p|X||Y|,
\end{aligned}
$$

where we used $\nu \leq \delta / 4$. Finally, (46) follows from (50) and (53).

5.2. Inheritance of the pair condition in neighborhoods of jumbled graphs. Lemma 21 asserts that the pair condition of a dense graph (i.e., $p=1$ ) is inherited on neighborhoods of a (possibly sparse) sufficiently jumbled graph. More precisely, suppose that $B=\left(X_{1} \cup X_{2}, E_{B}\right)$ is a dense bipartite graph that has property $\operatorname{PAIR}(\varrho, 1, \mu)$ for some constant $\varrho>0$ and sufficiently small $\mu>0$. Furthermore, let $\Gamma=\left(U \dot{\cup} V, E_{\Gamma}\right)$ be a bipartite $\left(p, \gamma p^{2} n\right)$-jumbled graph with $X_{1}, X_{2} \subseteq U$. Lemma 21 states that if $\gamma$ and $\mu$ are sufficiently small, then for most vertices $v \in V$ the pair condition $\operatorname{PAIR}(\varrho, 1, \nu)$ is inherited on the subgraph of $B$ induced on the neighborhood of $v$ in $\Gamma$.

Lemma 21. For every $\varrho_{0}>0$ and $\nu>0$ there exists $\mu>0$ such that for every $\eta>0$ there exist $\gamma>0$ and $n_{0}$ such that for every $n \geq n_{0}$ the following holds.

Suppose

(i) $\Gamma=\left(U \dot{\cup} V, E_{\Gamma}\right)$ is a bipartite $\left(p, \gamma p^{2} n\right)$-jumbled graph with $p \geq 1 / \sqrt{n}$,

(ii) $X_{1}, X_{2} \subseteq U$ and $Y \subseteq V$ with $\left|X_{1}\right|,\left|X_{2}\right|,|Y| \geq \eta n$, and

(iii) $B=\left(X_{1} \cup X_{2}, E_{B}\right)$ is an arbitrary bipartite graph.

Then the following statements hold:

(a) if $\left(X_{1}, X_{2}\right)_{B}$ satisfies $\operatorname{PAIR}(\varrho, 1, \mu)$ for some $\varrho$ with $\varrho_{0} \leq \varrho \leq 1$, then for all but at most $\nu|Y|$ vertices $y \in Y$ the pair $\left(N_{\Gamma}\left(y, X_{1}\right), N_{\Gamma}\left(y, X_{2}\right)\right)_{B}$ satisfies $\operatorname{PAIR}(\varrho, 1, \nu)$

(b) if $\left(X_{1}, X_{2}\right)_{B}$ satisfies $\operatorname{DISC}(\varrho, 1, \mu)$ for some $\varrho$ with $\varrho_{0} \leq \varrho \leq 1$, then for all but at most $\nu|Y|$ vertices $y \in Y$ the pair $\left(N_{\Gamma}\left(y, X_{1}\right), N_{\Gamma}\left(y, X_{2}\right)\right)_{B}$ satisfies $\operatorname{DISC}(\varrho, 1, \nu)$.

¿From the equivalence of DISC and PAIR for dense graphs (see Theorem 7), we infer that statements $(a)$ and $(b)$ are equivalent, and for the proof of Lemma 21 it suffices to verify only one of them. We will apply part $(b)$ of Lemma 21 in the proof of Lemma 9(a) (see Section 5.4 below). 
5.3. DISC implies PAIR. The proof of Lemma $9(a)$ is based on part $(b)$ of Lemma 21 and we briefly outline the main ideas below.

We will study an auxiliary graph with vertex set $X$ and edges corresponding to pairs $\left\{x, x^{\prime}\right\}$ for which $\left|\operatorname{deg}_{G}\left(x, x^{\prime}, Y\right)-q^{2}\right| Y||>\xi q^{2}|Y|$ for some constant $\xi$ depending only on $\delta$. We split those edges into two sets depending on whether $\operatorname{deg}_{G}\left(x, x^{\prime}, Y\right)>(1+\xi) q^{2}|Y|$ or $\operatorname{deg}_{G}\left(x, x^{\prime}, Y\right)<(1-\xi) q^{2}|Y|$ and call the resulting graphs $B^{+}$and $B^{-}$. The assumption that Lemma $9(a)$ fails implies that at least one of the graphs $B^{+}$or $B^{-}$has $\varrho\left(\begin{array}{c}|X| \\ 2\end{array}\right)$ edges for some $\varrho$ depending on $\alpha$ and $\delta$. Suppose $e\left(B^{+}\right)=\varrho\left(\begin{array}{c}|X| \\ 2\end{array}\right)$ (the argument for the other case is very similar). Applying Szemerédi's regularity lemma (or a weaker version ensuring just one pair with the discrepancy property (see Fact 22 below) to $B^{+}$shows that there exist subsets $X_{1}$ and $X_{2} \subseteq X$ such that $\left(X_{1}, X_{2}\right)_{B}$ satisfies $\operatorname{DISC}(\varrho, 1, \mu)$ for some small constant $0<\mu \ll \xi$ and, hence, we can apply part $(b)$ of Lemma 21 to $B=B^{+}\left[X_{1}, X_{2}\right]$ and $\Gamma$. Since $\left|N_{G}\left(y, X_{i}\right)\right| \geq(\alpha-\varepsilon) N_{\Gamma}\left(y, X_{i}\right)$ for most $y \in Y$ and $i=1,2$, the conclusion of part (b) of Lemma 21 yields good estimates on $e_{B}\left(N_{G}\left(y, X_{1}\right), N_{G}\left(y, X_{2}\right)\right)$ for most $y \in Y$. Based on this and the right choice of constants we will be able to show that the number of triples $\left(x_{1}, x_{2}, y\right) \in X_{1} \times X_{2} \times Y$ with $x_{1} \in N_{G}\left(y, X_{1}\right), x_{2} \in$ $N_{G}\left(y, X_{2}\right)$, and $\left\{x_{1}, x_{2}\right\} \in E(B)$ is bounded from above by $(1+\xi) q^{2} e(B)|Y|$. On the other hand, the definition of $B^{+}$implies that there are more than $(1+\xi) q^{2} e(B)|Y|$ such triples, which gives the desired contradiction. In the proof we will use the following fact, which asserts that every dense graph contains a pair of linear size that satisfies the discrepancy property.

Fact 22. Suppose $\varrho_{0}>0, \mu>0$, and $B=\left(X, E_{B}\right)$ is a graph with $\left|E_{B}\right| \geq \varrho_{0}\left(\begin{array}{c}|X| \\ 2\end{array}\right)$. Then there exist disjoint subsets $X_{1}, X_{2} \subseteq X$ such that

(a) $\left(X_{1}, X_{2}\right)_{B}$ satisfies $\operatorname{DISC}(\varrho, 1, \mu)$ for some $\varrho \geq \varrho_{0}$ and

(b) $\left|X_{1}\right|,\left|X_{2}\right| \geq \zeta$ n for $\zeta=\varrho_{0}^{100 / \mu^{2}} / 4$.

Proof. Fact 22 follows from [22, Theorem 1.1] applied with $d=\varrho_{0}$ and $\varepsilon=3 \mu / 4$.

Proof of Lemma 9 part $(a)$. First we define all constants required in this proof. Let $\alpha>0$ and $\delta>0$ be given. We set

$$
\xi=\frac{\delta}{6}
$$

and for the application of Lemma 21 we define

$$
\varrho_{0}=\frac{\delta}{50}
$$

and

$$
\nu=\frac{\alpha^{2} \xi \varrho_{0}}{64} .
$$

For this choice of $\varrho_{0}$ and $\nu$ Lemma 21 ensures the existence of some constant $\mu>0$. Without loss of generality we may assume that

$$
\mu \leq \frac{\xi \varrho_{0}}{4}
$$

Preparing for an application of Fact 22 we set

$$
\zeta=\frac{\varrho_{0}^{100 / \mu^{2}}}{4}
$$


and for Lemma 9 we define $\varepsilon=\varepsilon(\alpha, \delta)$ by

$$
\varepsilon=\min \left\{\frac{\alpha \delta^{2}}{36},\left(\frac{\alpha^{3} \xi \varrho_{0} \zeta}{64}\right)^{2}\right\} .
$$

Following the quantification of Lemma 9, we are given some $\eta>0$. We set

$$
\eta_{\mathrm{L} .21}=\eta \zeta .
$$

For this choice of $\eta_{\mathrm{L} .21}$ Lemma 21 yields $\gamma_{\mathrm{L} .21}>0$ and for Lemma 9 we set

$$
\gamma=\min \left\{\gamma_{\mathrm{L} .21}, \eta \sqrt{\delta / 3}, \alpha \sqrt{\xi \varrho_{0} \eta \eta_{\mathrm{L} .21} / 24}\right\}
$$

Finally, let $n$ be sufficiently large and suppose $p \geq 1 / \sqrt{n}$.

After we fixed all constants involved in the proof of Lemma 9 consider bipartite graphs $\Gamma=\left(U \dot{\cup} V, E_{\Gamma}\right)$ and $G=\left(U \dot{\cup} V, E_{G}\right)$ and sets $X \subseteq U$ and $Y \subseteq V$ that satisfy the assumptions of Lemma 9 . In particular, $(X, Y)_{G}$ satisfies $\operatorname{DISC}(q, p, \varepsilon)$ for some $q \geq \alpha p$. We want to infer that $(X, Y)_{G}$ satisfies $\operatorname{PAIR}(q, p, \delta)$, i.e., we have to verify that $(X, Y)_{G}$ satisfies (2) and (3) in Definition 6 .

Verifying (2) for $(X, Y)_{G}$. As we will see, this is a simple consequence of the given discrepancy property of $(X, Y)_{G}$ and the jumbledness of $\Gamma$. First we note that all but at most $\delta|X| / 6$ vertices $x \in X$ satisfy

$$
\left|\operatorname{deg}_{G}(x, Y)-q\right| Y|| \leq \frac{\delta}{3} q|Y|
$$

Otherwise there exists a set $X^{\prime} \subseteq X$ with $\left|X^{\prime}\right| \geq \delta|X| / 12$ such that for all $x \in X^{\prime}$ either $\operatorname{deg}_{G}(x, Y)>(1+\delta / 3) q|Y|$ or $\operatorname{deg}_{G}(x, Y)<(1-\delta / 3) q|Y|$. In either case, we would face

$$
\left|e\left(X^{\prime}, Y\right)-q\right| X^{\prime}|| Y||>\frac{\delta^{2}}{36} q|X||Y| \geq \frac{\delta^{2} \alpha}{36} p|X||Y| \stackrel{(59)}{\geq} \varepsilon p|X||Y|,
$$

which would contradict the assumption that $(X, Y)_{G}$ satisfies $\operatorname{DISC}(q, p, \varepsilon)$.

Fact 13 implies that the number of vertices $x \in X$ with $\operatorname{deg}_{\Gamma}(x, Y) \geq 2 p|Y|$ is bounded from above by $\gamma^{2} p^{2} n^{2} /|Y|$. Consequently,

$$
\sum_{x \in X}\left|\operatorname{deg}_{G}(x, Y)-q\right| Y|| \leq|X| \cdot \frac{\delta}{3} q|Y|+\frac{\delta}{6}|X| \cdot 2 p|Y|+\gamma^{2} p^{2} \frac{n^{2}}{|Y|} \cdot|Y| \leq \delta p|X||Y|,
$$

where the last inequality follows from $q \leq p$, the choice of $\gamma$ in (61) and assumption (iii) of Lemma 9.

Verifying (3) for $(X, Y)_{G}$. For the proof of (3) we proceed by contradiction. We consider the "bad" pairs $\left(x, x^{\prime}\right) \in X \times X$ for which $\operatorname{deg}_{G}\left(x, x^{\prime}, Y\right)$ deviates substantially from $q^{2}|Y|$. First we consider the following sets of bad pairs

$$
\begin{aligned}
& \mathcal{B}_{1}=\left\{\left(x, x^{\prime}\right) \in X \times X: \operatorname{deg}_{\Gamma}(x, Y)>2 p|Y|\right\}, \\
& \mathcal{B}_{2}=\left\{\left(x, x^{\prime}\right) \in(X \times X) \backslash \mathcal{B}_{1}: \operatorname{deg}_{\Gamma}\left(x, x^{\prime}, Y\right)>4 p^{2}|Y|\right\} .
\end{aligned}
$$

¿From the $\left(p, \gamma p^{2} n\right)$-jumbledness of $\Gamma$, the choice $\gamma$ in (61), and assumption (iii) of Lemma 9 combined with Fact 13, it follows that

$$
\left|\mathcal{B}_{1}\right| \leq \gamma^{2} p^{2} \frac{n^{2}}{|Y|} \cdot|X| \leq \frac{\delta}{3} p^{2}|X|^{2}
$$

For an upper bound on $\left|\mathcal{B}_{2}\right|$ we consider a vertex $x \in X$ with $\operatorname{deg}_{\Gamma}(x, Y) \leq 2 p|Y|$. Applying Fact 13 to a superset of $N_{\Gamma}(x, Y)$ with $2 p|Y|$ elements implies that there 
are at most $\gamma^{2} p n^{2} /(2|Y|)$ vertices $x^{\prime}$ such that $\operatorname{deg}_{\Gamma}\left(x, x^{\prime}, Y\right)>4 p^{2}|Y|$. Consequently,

$$
\left|\mathcal{B}_{2}\right| \leq|X| \cdot \gamma^{2} p \frac{n^{2}}{2|Y|} \leq \frac{\delta}{6} p|X|^{2} .
$$

Combining those estimates on $\left|\mathcal{B}_{1}\right|$ and $\left|\mathcal{B}_{2}\right|$ with the assumption that (3) fails, we infer

$$
\begin{aligned}
\sum_{\left(x, x^{\prime}\right) \notin \mathcal{B}_{1} \cup \mathcal{B}_{2}}\left|\operatorname{deg}_{G}\left(x, x^{\prime}, Y\right)-q^{2}\right| Y|| & >\delta p^{2}|X|^{2}|Y|-\left|\mathcal{B}_{1}\right| \cdot|Y|-\left|\mathcal{B}_{2}\right| \cdot 2 p|Y| \\
& \geq \frac{\delta}{3} p^{2}|X|^{2}|Y| .
\end{aligned}
$$

In other words we have just shown that ignoring the contribution of pairs $\left(x, x^{\prime}\right) \in$ $\mathcal{B}_{1} \cup \mathcal{B}_{2}$ does not affect in an essential way the property drawn from the assumption that (3) fails.

Moreover, note that the total contribution of pairs $\left(x, x^{\prime}\right) \notin \mathcal{B}_{1} \cup \mathcal{B}_{2}$ with $\left|\operatorname{deg}_{G}\left(x, x^{\prime}, Y\right)-q^{2}\right| Y|| \leq \delta q^{2}|Y| / 6$ to the sum considered in (62) is at most

$$
\frac{\delta}{6} q^{2}|X|^{2}|Y| \leq \frac{\delta}{6} p^{2}|X|^{2}|Y|
$$

Since, furthermore, for all $\left(x, x^{\prime}\right) \notin \mathcal{B}_{1} \cup \mathcal{B}_{2}$ we have

$$
\left|\operatorname{deg}_{G}\left(x, x^{\prime}, Y\right)-q^{2}\right| Y|| \leq \max \left\{q^{2}|Y|,\left(4 p^{2}-q^{2}\right)|Y|\right\} \leq 4 p^{2}|Y|,
$$

there are at least $\delta|X|^{2} / 24$ pairs $\left(x, x^{\prime}\right) \in(X \times X) \backslash\left(\mathcal{B}_{1} \cup \mathcal{B}_{2}\right)$ such that

$$
\left|\operatorname{deg}_{G}\left(x, x^{\prime}, Y\right)-q^{2}\right| Y||>\frac{\delta}{6} q^{2}|Y| \stackrel{(54)}{=} \xi q^{2}|Y| \text {. }
$$

Next we consider the graphs $B^{+}$and $B^{-}$with vertex set $X$ and edges corresponding to those pairs defined by

$$
E\left(B^{+}\right)=\left\{\left\{x, x^{\prime}\right\}:(1+\xi) q^{2}|Y|<\operatorname{deg}_{G}\left(x, x^{\prime}, Y\right) \leq 4 p^{2}|Y| \text { and } x \neq x^{\prime}\right\}
$$

and

$$
E\left(B^{-}\right)=\left\{\left\{x, x^{\prime}\right\}: \operatorname{deg}_{G}\left(x, x^{\prime}, Y\right)<(1-\xi) q^{2}|Y| \text { and } x \neq x^{\prime}\right\} .
$$

Since there are at least $\delta|X|^{2} / 24$ ordered pairs $\left(x, x^{\prime}\right)$ for which (63) holds, for sufficiently large $n$ we have

$$
\max \left\{e\left(B^{+}\right), e\left(B^{-}\right)\right\} \geq \frac{\delta}{96}|X|^{2}-|X| \stackrel{(55)}{\geq} \varrho_{0}\left(\begin{array}{c}
|X| \\
2
\end{array}\right) .
$$

Below we assume $e\left(B^{+}\right) \geq \varrho_{0}\left(\begin{array}{c}|X| \\ 2\end{array}\right)$ and remark that the argument for the case $e\left(B^{-}\right) \geq \varrho_{0}\left(\begin{array}{c}|X| \\ 2\end{array}\right)$ follows the same lines.

Fact 22, combined with the choice of $\zeta$ in (58), gives that there exist disjoint subsets $X_{1}, X_{2} \subseteq X$ such that $\left(X_{1}, X_{2}\right)_{B^{+}}$satisfies $\operatorname{DISC}(\varrho, 1, \mu)$ for some $\varrho \geq \varrho_{0}$ and $\left|X_{1}\right|,\left|X_{2}\right| \geq \zeta|X| \geq \zeta \eta n=\eta_{\mathrm{L} .21} n$ (see (60)).

Hence, it follows from part $(b)$ of Lemma 21 that for all but at most $\nu|Y|$ vertices $y \in Y$ we have that

$$
\left(N_{\Gamma}\left(y, X_{1}\right), N_{\Gamma}\left(y, X_{2}\right)\right)_{B^{+}} \text {satisfies } \operatorname{DISC}(\varrho, 1, \nu) .
$$

Based on (65) we obtain estimates on the number of triplets $\left(x_{1}, x_{2}, y\right)$ from $X_{1} \times X_{2} \times Y$ such that $\left\{x_{1}, x_{2}\right\}$ is an edge in $B^{+}$contained in the neighborhood of 
$y$ in $G$. Let

$$
\begin{aligned}
\mathcal{T}=\left\{\left(x_{1}, x_{2}, y\right) \in\right. & X_{1} \times X_{2} \times Y: \\
& \left.x_{1} \in N_{G}\left(y, X_{1}\right), x_{2} \in N_{G}\left(y, X_{2}\right), \text { and }\left\{x_{1}, x_{2}\right\} \in E\left(B^{+}\right)\right\} .
\end{aligned}
$$

From the definition of $B^{+}$in (64) and the fact that $\left(X_{1}, X_{2}\right)_{B^{+}}$satisfies $\operatorname{DISC}(\varrho, 1, \mu)$ we infer

$$
\begin{aligned}
|\mathcal{T}|>(1+\xi) q^{2}|Y| \cdot e_{B^{+}}\left(X_{1}, X_{2}\right) \geq(1+\xi) q^{2}|Y| \cdot(\varrho-\mu)\left|X_{1}\right|\left|X_{2}\right| & \stackrel{(57)}{\geq}\left(1+\frac{\xi}{2}\right) \varrho q^{2}\left|X_{1}\right|\left|X_{2}\right||Y| .
\end{aligned}
$$

On the other hand,

$$
|\mathcal{T}|=\sum_{y \in Y} e_{B^{+}}\left(N_{G}\left(y, X_{1}\right), N_{G}\left(y, X_{2}\right)\right) .
$$

Let $Y^{\prime}=\left\{y \in Y: \operatorname{deg}_{\Gamma}\left(y, X_{i}\right) \leq 2 p\left|X_{i}\right|\right.$ for $\left.i=1,2\right\}$. It follows from (65) that for all but at most $\nu|Y|$ vertices $y \in Y^{\prime}$ we have

$$
\begin{aligned}
e_{B^{+}}\left(N_{G}\left(y, X_{1}\right), N_{G}\left(y, X_{2}\right)\right) & \leq \varrho\left|N_{G}\left(y, X_{1}\right)\right|\left|N_{G}\left(y, X_{2}\right)\right|+\nu\left|N_{\Gamma}\left(y, X_{1}\right)\right|\left|N_{\Gamma}\left(y, X_{2}\right)\right| \\
& \leq \varrho \operatorname{deg}_{G}\left(y, X_{1}\right) \operatorname{deg}_{G}\left(y, X_{2}\right)+4 \nu p^{2}\left|X_{1}\right|\left|X_{2}\right| .
\end{aligned}
$$

Fact 13 gives

$$
\left|Y \backslash Y^{\prime}\right| \leq \gamma^{2} p^{2} \frac{n^{2}}{\left|X_{1}\right|}+\gamma^{2} p^{2} \frac{n^{2}}{\left|X_{2}\right|}
$$

Consequently,

$$
\begin{aligned}
|\mathcal{T}| \leq \sum_{y \in Y^{\prime}}\left(\varrho \operatorname{deg}_{G}(\right. & \left.\left.y, X_{1}\right) \operatorname{deg}_{G}\left(y, X_{2}\right)+4 \nu p^{2}\left|X_{1}\right|\left|X_{2}\right|\right) \\
& +\nu|Y| \cdot 4 p^{2}\left|X_{1}\right|\left|X_{2}\right|+\left(\gamma^{2} p^{2} \frac{n^{2}}{\left|X_{1}\right|}+\gamma^{2} p^{2} \frac{n^{2}}{\left|X_{2}\right|}\right) \cdot\left|X_{1}\right|\left|X_{2}\right|
\end{aligned}
$$

and the choices of $\nu$ in (56) and $\gamma$ in (61), combined with $\left|X_{1}\right|,\left|X_{2}\right| \geq \eta_{\mathrm{L} .21} n$ and $Y \geq \eta n$ and $\varrho \geq \varrho_{0}$, yield

$$
|\mathcal{T}| \leq \varrho \sum_{y \in Y^{\prime}} \operatorname{deg}_{G}\left(y, X_{1}\right) \operatorname{deg}_{G}\left(y, X_{2}\right)+\frac{\xi}{4} \varrho q^{2}\left|X_{1}\right|\left|X_{2}\right||Y| .
$$

Finally, we appeal to the assumption of Lemma $9(a)$ stating that $(X, Y)_{G}$ satisfies $\operatorname{DISC}(q, p, \varepsilon)$. As $\left|X_{1}\right|,\left|X_{2}\right| \geq \zeta|X|$ and $q \geq \alpha p$ we have for $i=1$ and 2

$$
\left|\left\{y \in Y: \operatorname{deg}_{G}\left(y, X_{i}\right)>(1+\sqrt{\varepsilon}) q\left|X_{i}\right|\right\}\right| \leq \frac{\varepsilon p|X||Y|}{\sqrt{\varepsilon} q\left|X_{i}\right|} \leq \frac{\sqrt{\varepsilon}}{\alpha \zeta}|Y| \stackrel{(59)}{\leq} \frac{\xi \alpha^{2} \varrho}{64}|Y| .
$$

Since $\sqrt{\varepsilon} \leq \xi / 24$ we can further bound the right-hand side of (67) and obtain

$$
\begin{array}{r}
|\mathcal{T}| \leq \varrho|Y| \cdot(1+\sqrt{\varepsilon})^{2} q^{2}\left|X_{1}\right|\left|X_{2}\right|+\frac{\xi \alpha^{2} \varrho}{32}|Y| \cdot 4 p^{2}\left|X_{1}\right|\left|X_{2}\right|+\frac{\xi}{4} \varrho q^{2}\left|X_{1}\right|\left|X_{2}\right||Y| \\
\leq\left(1+\frac{\xi}{2}\right) \varrho q^{2}\left|X_{1}\right|\left|X_{2}\right||Y|,
\end{array}
$$

which contradicts (66). 
5.4. Proof of Lemma 21. Recall that it suffices to prove part ( $a$ ) of Lemma 21 and then part $(b)$ follows from Theorem 7.

Proof of Lemma 21. Let $\varrho_{0}$ and $\nu$ be given. We fix an auxiliary constant

$$
\nu^{\prime}=\frac{\nu^{2}}{100}
$$

and let $\mu^{\prime \prime}>0$ be given by Lemma 12 applied with $\nu^{\prime \prime}=\nu^{\prime} / 4$. Moreover, fix $\mu^{\prime}$ and $\xi$ with $0<\mu^{\prime} \leq \mu^{\prime \prime} / 2$ and $0<\xi<\min \left\{\mu^{\prime \prime} / 6, \nu^{\prime} / 4\right\}$ so that

$$
\frac{\left(1-\mu^{\prime}\right)^{2}}{1+\xi} \geq 1-\frac{\mu^{\prime \prime}}{2} \quad \text { and } \quad(1+\xi) \sqrt{1+\mu^{\prime}} \leq 1+\frac{\mu^{\prime \prime}}{2} .
$$

Next let $\mu$ be sufficiently small, so that

$$
\mu \leq \frac{\varrho_{0}^{4} \mu^{\prime}}{5}, \quad \frac{\sqrt{\mu}}{\varrho^{2}}(1+\mu)^{2}(1+\xi)^{2} \leq \frac{\mu^{\prime} \varrho^{4}}{5}, \text { and }(1+\mu)^{4}(1+\sqrt{\mu})^{2} \leq 1+\frac{\mu^{\prime}}{5} .
$$

For any given $\eta>0$, we let

$$
\gamma=\min \left\{\xi \eta \sqrt{\nu / 12}, \mu^{3 / 2} \eta \varrho_{0} / 2\right\} .
$$

Let $n$ be sufficiently large and suppose $p \geq 1 / \sqrt{n}$.

Suppose the bipartite graph $\Gamma=\left(U \dot{U} V, E_{\Gamma}\right)$, the sets $X_{1}, X_{2} \subseteq U$, and $Y \subseteq$ $V$, and the bipartite graph $B=\left(X_{1} \cup X_{2}, E_{B}\right)$ satisfy assumptions $(i)-($ iii $)$ of Lemma 21, Moreover, suppose that $\left(X_{1}, X_{2}\right)_{B}$ satisfies $\operatorname{PAIR}(\varrho, 1, \mu)$ for some $\varrho \geq$ $\varrho_{0}$. We have to show that for all but $\nu|Y|$ vertices $y \in Y$ the pair condition is inherited in the subgraph of $B$ induced on the neighborhoods of $y$ in $\Gamma$, i.e., $\left(N_{\Gamma}\left(y, X_{1}\right), N_{\Gamma}\left(y, X_{2}\right)\right)_{B}$ satisfies $\operatorname{PAIR}(\varrho, 1, \nu)$.

In the first step we exclude all vertices $y \in Y$ for which $\operatorname{deg}_{\Gamma}\left(y, X_{1}\right) \operatorname{or}_{\operatorname{deg}_{\Gamma}}\left(y, X_{2}\right)$ deviates substantially from its "expectation." For $i=1$ and 2 we set

$$
Y_{i}^{*}=\left\{y \in Y:\left|\operatorname{deg}_{\Gamma}\left(y, X_{i}\right)-p\right| X_{i}||>\xi p\left|X_{i}\right|\right\} \text { and } Y^{*}=Y_{1}^{*} \cup Y_{2}^{*} .
$$

Since $\Gamma$ is $\left(p, \gamma p^{2} n\right)$-jumbled and $\left|X_{1}\right|,\left|X_{2}\right|,|Y| \geq \eta n$, Fact 13 yields

$$
\left|Y^{*}\right| \leq\left|Y_{1}^{*}\right|+\left|Y_{2}^{*}\right| \leq \frac{2 \gamma^{2} p^{4} n^{2}}{\xi^{2} p^{2}\left|X_{1}\right|}+\frac{2 \gamma^{2} p^{4} n^{2}}{\xi^{2} p^{2}\left|X_{2}\right|} \leq \frac{4 \gamma^{2} p^{2}|Y|}{\xi^{2} \eta^{2}} .
$$

Since $p \leq 1$, the choice of $\gamma$ in (71) yields

$$
\left|Y^{*}\right| \leq \frac{\nu}{3}|Y|
$$

For the rest of the proof we will ignore the vertices from $Y^{*}$ and they will be included in the set of $\nu|Y|$ exceptional vertices.

Next we study the triplets $\left(x_{1}, x_{2}, y\right) \in X_{1} \times X_{2} \times\left(Y \backslash Y^{*}\right)$ with $\left\{x_{1}, x_{2}\right\}$ being an edge of $B$ and $\left\{x_{1}, y\right\}$ and $\left\{x_{2}, y\right\}$ being present in $\Gamma$. We set

$$
Y^{\prime}=Y \backslash Y^{*}
$$

and define

$$
t_{1,1}=\sum_{y \in Y^{\prime}} \sum_{x_{1} \in N_{\Gamma}\left(y, X_{1}\right)} \operatorname{deg}_{B}\left(x_{1}, N_{\Gamma}\left(y, X_{2}\right)\right)
$$


as well as

$$
\begin{aligned}
t_{1,2} & =\sum_{y \in Y^{\prime}} \sum_{x_{1} \in N_{\Gamma}\left(y, X_{1}\right)} \operatorname{deg}_{B}^{2}\left(x_{1}, N_{\Gamma}\left(y, X_{2}\right)\right) \\
t_{2,1} & =\sum_{y \in Y^{\prime}} \sum_{x_{2} \in N_{\Gamma}\left(y, X_{2}\right)} \operatorname{deg}_{B}^{2}\left(x_{2}, N_{\Gamma}\left(y, X_{1}\right)\right)
\end{aligned}
$$

and

$$
t_{2,2}=\sum_{y \in Y^{\prime}} \sum_{x_{1} \in N_{\Gamma}\left(y, X_{1}\right)} \sum_{x_{1}^{\prime} \in N_{\Gamma}\left(y, X_{1}\right)} \operatorname{deg}_{B}^{2}\left(x_{1}, x_{1}^{\prime}, N_{\Gamma}\left(y, X_{2}\right)\right) .
$$

Below we will verify the following bounds on $t_{1,1}$ and $t_{2,2}$

$$
t_{1,1} \geq\left(1-\mu^{\prime}\right) \varrho p^{2}\left|X_{1}\right|\left|X_{2}\right|\left|Y^{\prime}\right|
$$

and

$$
t_{2,2} \leq\left(1+\mu^{\prime}\right) \varrho^{4} p^{4}\left|X_{1}\right|^{2}\left|X_{2}\right|^{2}\left|Y^{\prime}\right|
$$

Before we prove (73) and (74) we deduce the conclusion of Lemma 21 from these estimates.

Since $\left|N_{\Gamma}\left(y, X_{1}\right)-p\right| X_{1}|| \leq \xi p\left|X_{1}\right|$ for all $y \in Y^{\prime}$ the number of summands $m$ considered in $t_{1,1}$ is bounded by $\left|Y^{\prime}\right|(1+\xi) p\left|X_{1}\right|$. Hence, it follows from (73) combined with the Cauchy-Schwarz inequality $\sum_{i=1}^{m} a_{i}^{2} \geq\left(\sum_{i=1}^{m} a_{i}\right)^{2} / m$ that

$$
t_{1,2} \geq \frac{\left(1-\mu^{\prime}\right)^{2}}{1+\xi} \varrho^{2} p^{3}\left|X_{1}\right|\left|X_{2}\right|^{2}\left|Y^{\prime}\right| \stackrel{(69)}{\geq}\left(1-\mu^{\prime \prime} / 2\right) \varrho^{2} p^{3}\left|X_{1}\right|\left|X_{2}\right|^{2}\left|Y^{\prime}\right| \text {. }
$$

Moreover, owing to the identity

$$
t_{1,1}=\sum_{y \in Y^{\prime}} \sum_{x_{2} \in N_{\Gamma}\left(y, X_{2}\right)} \operatorname{deg}_{B}\left(x_{2}, N_{\Gamma}\left(y, X_{1}\right)\right)
$$

the same argument gives

$$
t_{2,1} \geq\left(1-\mu^{\prime \prime} / 2\right) \varrho^{2} p^{3}\left|X_{1}\right|^{2}\left|X_{2}\right|\left|Y^{\prime}\right|
$$

Similarly, since we can rewrite $t_{2,1}$ as

$$
t_{2,1}=\sum_{y \in Y^{\prime}} \sum_{x_{1} \in N_{\Gamma}\left(y, X_{1}\right)} \sum_{x_{1}^{\prime} \in N_{\Gamma}\left(y, X_{1}\right)} \operatorname{deg}_{B}\left(x_{1}, x_{1}^{\prime}, N_{\Gamma}\left(y, X_{2}\right)\right)
$$

the bound in (74) combined with the Cauchy-Schwarz inequality yields

$$
t_{2,1} \leq(1+\xi) \sqrt{1+\mu^{\prime}} \varrho^{2} p^{3}\left|X_{1}\right|^{2}\left|X_{2}\right|\left|Y^{\prime}\right| \stackrel{(69)}{\leq}\left(1+\mu^{\prime \prime} / 2\right) \varrho^{2} p^{3}\left|X_{1}\right|^{2}\left|X_{2}\right|\left|Y^{\prime}\right| .
$$

In a very similar way we obtain

$$
t_{1,2} \leq\left(1+\mu^{\prime \prime} / 2\right) \varrho^{2} p^{3}\left|X_{1}\right|\left|X_{2}\right|^{2}\left|Y^{\prime}\right|
$$

Summarizing (75)-(78), we showed that (73) and (74) yield

$$
\left.\left.\left|t_{1,2}-\varrho^{2} p^{3}\right| X_{1}|| X_{2}\right|^{2}\left|Y^{\prime}\right|\left|\leq \frac{\mu^{\prime \prime}}{2} \varrho^{2} p^{3}\right| X_{1}|| X_{2}\right|^{2}\left|Y^{\prime}\right|
$$

and

$$
\left.\left.\left|t_{2,1}-\varrho^{2} p^{3}\right| X_{1}\right|^{2}\left|X_{2}\right|\left|Y^{\prime}\right|\left|\leq \frac{\mu^{\prime \prime}}{2} \varrho^{2} p^{3}\right| X_{1}\right|^{2}\left|X_{2}\right|\left|Y^{\prime}\right| .
$$

In particular, (73) gives

$$
t_{1,1} \geq\left(1-\mu^{\prime \prime}\right)(1+\xi) \varrho p^{2}\left|X_{1}\right|\left|X_{2}\right|\left|Y^{\prime}\right|
$$


and, similarly, (79) gives

$$
t_{1,2} \leq\left(1+\mu^{\prime \prime}\right)(1-\xi) \varrho^{2} p^{3}\left|X_{1}\right|\left|X_{2}\right|^{2}\left|Y^{\prime}\right| .
$$

Recalling the definition of $Y^{\prime}$, by Lemma 12 we see that those estimates yield

$$
\begin{aligned}
|| N_{B}\left(x_{1},\right. & \left.N_{\Gamma}\left(y, X_{2}\right)\right)|-\varrho| N_{\Gamma}\left(y, X_{2}\right)|| \\
& \leq|| N_{B}\left(x_{1}, N_{\Gamma}\left(y, X_{2}\right)\right)|-\varrho p| X_{2}||+\xi \varrho p\left|X_{2}\right| \\
& \leq\left(\frac{\nu^{\prime}}{4}+\xi\right) \varrho p\left|X_{2}\right| \leq \nu^{\prime}\left|N_{\Gamma}\left(y, X_{2}\right)\right|
\end{aligned}
$$

for all but at most $\nu^{\prime}(1+\xi) p\left|X_{1} \| Y^{\prime}\right|$ pairs $\left(y, x_{1}\right) \in Y \times X_{1}$ with $x_{1} \in N_{\Gamma}\left(y, X_{1}\right)$. Let $Y^{* *} \subseteq Y^{\prime}$ contain those vertices $y$ for which there are at least $\nu\left|N_{\Gamma}\left(y, X_{1}\right)\right| / 2$ vertices $x_{1} \in N_{\Gamma}\left(y, X_{1}\right)$ such that (81) fails. Since (81) fails for at most $\nu^{\prime}(1+\xi) p\left|X_{1}\right|\left|Y^{\prime}\right|$ pairs and each $y \in Y^{* *}$ is contained in at least $\nu\left|N_{\Gamma}\left(y, X_{1}\right)\right| / 2$ such pairs we have

$$
\left|Y^{* *}\right| \leq \frac{2 \nu^{\prime}(1+\xi) p\left|X_{1}\right|\left|Y^{\prime}\right|}{\nu(1-\xi) p\left|X_{1}\right|} \stackrel{(68)}{\leq} \frac{\nu}{3}|Y|
$$

Recalling that for $y \notin Y^{*} \cup Y^{* *}$ a "typical" vertex $x_{1} \in N_{\Gamma}\left(y, X_{1}\right)$ satisfies (81) and that there are at most $\nu\left|N_{\Gamma}\left(y, X_{1}\right)\right| / 2$ "atypical" vertices we infer

$$
\begin{aligned}
\sum_{x_{1} \in N_{\Gamma}\left(y, X_{1}\right)} & || N_{B}\left(x_{1}, N_{\Gamma}\left(y, X_{2}\right)\right)||-\varrho\left|N_{\Gamma}\left(y, X_{2}\right)\right| \mid \\
& \leq\left(\nu^{\prime}+\frac{\nu}{2}\right)\left|N_{\Gamma}\left(y, X_{1}\right)\right|\left|N_{\Gamma}\left(y, X_{2}\right)\right| \leq \nu\left|N_{\Gamma}\left(y, X_{1}\right)\right|\left|N_{\Gamma}\left(y, X_{2}\right)\right|,
\end{aligned}
$$

which verifies the first part of $\operatorname{PAIR}(\varrho, 1, \nu)$ (i.e., $(2))$ for $\left(N_{\Gamma}\left(y, X_{1}\right), N_{\Gamma}\left(y, X_{2}\right)\right)_{B}$ for all $y \in Y \backslash\left(Y^{*} \cup Y^{* *}\right)$.

Next we deduce the second part of $\operatorname{PAIR}(\varrho, 1, \nu)$ (i.e., (3)) for "most" vertices $y \in Y \backslash\left(Y^{*} \cup Y^{* *}\right)$ from (80) and (74) in a very similar manner.

In fact, (80), (74), and $\xi \leq \mu^{\prime \prime} / 6$ imply

$$
t_{2,1} \geq\left(1-\mu^{\prime \prime}\right)(1+\xi)^{2} \varrho^{2} p^{3}\left|X_{1}\right|^{2}\left|X_{2}\right|\left|Y^{\prime}\right|
$$

and

$$
t_{2,2} \leq\left(1+\mu^{\prime \prime}\right)(1-\xi)^{2} \varrho^{4} p^{4}\left|X_{1}\right|^{2}\left|X_{2}\right|^{2}\left|Y^{\prime}\right| .
$$

By the definition of $Y^{\prime}$, Lemma 12 yields

$$
\begin{aligned}
|| N_{B}\left(x_{1},\right. & \left.x_{1}^{\prime}, N_{\Gamma}\left(y, X_{2}\right)\right)\left|-\varrho^{2}\right| N_{\Gamma}\left(y, X_{2}\right)|| \\
& \leq|| N_{B}\left(x_{1}, x_{1}^{\prime}, N_{\Gamma}\left(y, X_{2}\right)\right)\left|-\varrho^{2} p\right| X_{2}||+\xi \varrho^{2} p\left|X_{2}\right| \\
& \leq\left(\frac{\nu^{\prime}}{4}+\xi\right) \varrho^{2} p\left|X_{2}\right| \leq \nu^{\prime}\left|N_{\Gamma}\left(y, X_{2}\right)\right|
\end{aligned}
$$

for all but at most $\nu^{\prime}(1+\xi)^{2} p^{2}\left|X_{1}\right|^{2}\left|Y^{\prime}\right|$ triplets $\left(y, x_{1}, x_{1}^{\prime}\right) \in Y \times X_{1} \times X_{1}$ with $x_{1}, x_{1}^{\prime} \in N_{\Gamma}\left(y, X_{1}\right)$. Let $Y^{* * *} \subseteq Y^{\prime}$ contain those vertices $y$ for which there are at least $\nu\left|N_{\Gamma}\left(y, X_{1}\right)\right|^{2} / 2$ vertices $x_{1}, x_{1}^{\prime} \in N_{\Gamma}\left(y, X_{1}\right)$ such that (84) fails. It follows from the definition of $Y^{* * *}$ that

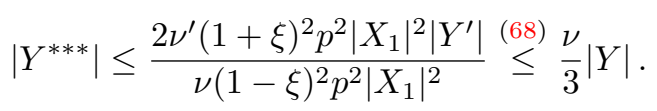


Finally, we note that for all vertices $y \in Y \backslash\left(Y^{*} \cup Y^{* * *}\right)$ we have

$$
\begin{aligned}
& \sum_{x_{1} \in N_{\Gamma}\left(y, X_{1}\right)} \sum_{x_{1}^{\prime} \in N_{\Gamma}\left(y, X_{1}\right)}|| N_{B}\left(x_{1}, x_{1}^{\prime}, N_{\Gamma}\left(y, X_{2}\right)\right)\left|-\varrho^{2}\right| N_{\Gamma}\left(y, X_{2}\right)|| \\
& \leq\left(\nu^{\prime}+\frac{\nu}{2}\right)\left|N_{\Gamma}\left(y, X_{1}\right)\right|^{2}\left|N_{\Gamma}\left(y, X_{2}\right)\right| \leq \nu\left|N_{\Gamma}\left(y, X_{1}\right)\right|^{2}\left|N_{\Gamma}\left(y, X_{2}\right)\right|,
\end{aligned}
$$

which verifies (3) for $\left(N_{\Gamma}\left(y, X_{1}\right), N_{\Gamma}\left(y, X_{2}\right)\right)_{B}$ for all $y \in Y \backslash\left(Y^{*} \cup Y^{* * *}\right)$.

Inequalities (83) and (86) imply that for every $y \notin Y^{*} \cup Y^{* *} \cup Y^{* * *}$ the pair $\left(N_{\Gamma}\left(y, X_{1}\right), N_{\Gamma}\left(y, X_{2}\right)\right)_{B}$ satisfies $\operatorname{PAIR}(\varrho, 1, \nu)$. Moreover, owing to (72), (82), and (85), we have

$$
\left|Y^{*} \cup Y^{* *} \cup Y^{* * *}\right| \leq \nu|Y| .
$$

This concludes the proof of part (a) of Lemma 21. It remains to verify (73) and (74).

Proof of (73). In order to verify (73) we have to estimate the number of triplets $\left(x_{1}, x_{2}, y\right) \in X_{1} \times X_{2} \times Y^{\prime}$ such that $\left\{x_{1}, x_{2}\right\}$ is an edge of $B$ and $x_{1}$ and $x_{2}$ are neighbors of $y$ in $\Gamma$.

We recall the assumption that $\left(X_{1}, X_{2}\right)_{B}$ satisfies $\operatorname{PAIR}(\varrho, 1, \mu)$ and, therefore,

$$
\sum_{x_{1} \in X_{1}}\left|\operatorname{deg}_{B}\left(x_{1}, X_{2}\right)-\varrho\right| X_{2}|| \leq \mu\left|X_{1}\right|\left|X_{2}\right| .
$$

Consequently,

$$
e_{B}\left(X_{1}, X_{2}\right) \geq(\varrho-\mu)\left|X_{1}\right|\left|X_{2}\right| .
$$

Moreover, owing to Fact 13 all but at most

$$
\frac{\gamma^{2} p^{2} n^{2}}{\mu^{2}\left|Y^{\prime}\right|} \leq \frac{\gamma^{2} p^{2} n^{2}}{\mu^{2}(1-\nu / 3)|Y|} \stackrel{(71)}{\leq} \mu\left|X_{1}\right|
$$

vertices $x_{1} \in X_{1}$ satisfy

$$
\operatorname{deg}_{\Gamma}\left(x_{1}, Y^{\prime}\right) \geq(1-\mu) p\left|Y^{\prime}\right| .
$$

Finally, for every vertex $x_{1}$ for which (89) holds another application of Fact 13 yields that

$$
\operatorname{deg}_{\Gamma}\left(x_{2}, N_{\Gamma}\left(x_{1}, Y^{\prime}\right)\right) \geq(1-\mu) p \operatorname{deg}_{\Gamma}\left(x_{1}, Y^{\prime}\right) \geq(1-\mu)^{2} p^{2}\left|Y^{\prime}\right|
$$

for all but at most

$$
\frac{\gamma^{2} p^{2} n^{2}}{\mu^{2} \operatorname{deg}_{\Gamma}\left(x_{1}, Y^{\prime}\right)} \leq \frac{\gamma^{2} p^{2} n^{2}}{\mu^{2} \cdot(1-\mu) p\left|Y^{\prime}\right|} \leq \frac{\gamma^{2} p^{2} n^{2}}{\mu^{2} \cdot(1-\mu)(1-\nu / 3) p|Y|} \stackrel{(71)}{\leq} \mu\left|X_{2}\right| .
$$

vertices $x_{2} \in X_{2}$. Summarizing (88)-(91), we infer that for all but at most $2 \mu\left|X_{1}\right|\left|X_{2}\right|$ pairs of vertices $\left(x_{1}, x_{2}\right) \in X_{1} \times X_{2}$ we have

$$
\operatorname{deg}_{\Gamma}\left(x_{1}, x_{2}, Y^{\prime}\right) \geq(1-\mu)^{2} p^{2}\left|Y^{\prime}\right| .
$$

Combining this with (87) yields

$$
\begin{aligned}
t_{1,1} & \geq\left(e_{B}\left(X_{1}, X_{2}\right)-2 \mu\left|X_{1}\right|\left|X_{2}\right|\right) \cdot(1-\mu)^{2} p^{2}\left|Y^{\prime}\right| \\
& \geq(\varrho-3 \mu)\left|X_{1}\right|\left|X_{2}\right| \cdot(1-\mu)^{2} p^{2}\left|Y^{\prime}\right| \stackrel{(70)}{\geq}\left(1-\mu^{\prime}\right) \varrho p^{2}\left|X_{1}\right|\left|X_{2}\right|\left|Y^{\prime}\right|,
\end{aligned}
$$

which concludes the proof of (73). 
Proof of (74). In order to verify (74) we will find an upper bound on the number of 5-tuples $\left(x_{1}, x_{1}^{\prime}, y, x_{2}, x_{2}^{\prime}\right)$ satisfying $y \in Y^{\prime}, x_{1}, x_{1}^{\prime} \in N_{\Gamma}\left(y, X_{1}\right), x_{2}, x_{2}^{\prime} \in N_{\Gamma}\left(y, X_{2}\right)$, with $x_{1}, x_{1}^{\prime}, x_{2}$, and $x_{2}^{\prime}$ spanning a cycle of length 4 in $B$.

First we appeal to the assumption that $\left(X_{1}, X_{2}\right)_{B}$ satisfies $\operatorname{PAIR}(\varrho, 1, \mu)$ and, hence,

$$
\sum_{x_{1} \in X_{1}} \sum_{x_{1}^{\prime} \in X_{1}}\left|\operatorname{deg}_{B}\left(x_{1}, x_{1}^{\prime}, X_{2}\right)-\varrho^{2}\right| X_{2}|| \leq \mu\left|X_{1}\right|^{2}\left|X_{2}\right| .
$$

Consequently, all but at most

$$
\frac{\sqrt{\mu}}{\varrho^{2}}\left|X_{1}\right|^{2}
$$

pairs $\left(x_{1}, x_{1}^{\prime}\right) \in X_{1} \times X_{1}$ satisfy

$$
\left|\operatorname{deg}_{B}\left(x_{1}, x_{1}^{\prime}, X_{2}\right)-\varrho^{2}\right| X_{2}|| \leq \sqrt{\mu} \varrho^{2}\left|X_{2}\right| .
$$

Three applications of Fact 13 yield the following estimates:

(A) all but at most

$$
\frac{2 \gamma^{2} p^{4} n^{2}}{\mu^{2} p^{2}\left|Y^{\prime}\right|} \stackrel{(71)}{\leq} \mu p^{2}\left|X_{1}\right|
$$

vertices $x_{1} \in X_{1}$ satisfy

$$
\left|\operatorname{deg}_{\Gamma}\left(x_{1}, Y^{\prime}\right)-p\right| Y^{\prime}|| \leq \mu p\left|Y^{\prime}\right| .
$$

(B) for every $x_{1} \in X_{1}$ satisfying (95), all but at most

$$
\frac{\gamma^{2} p^{4} n^{2}}{\mu^{2} p^{2}\left|N_{\Gamma}\left(x_{1}, Y^{\prime}\right)\right|} \stackrel{(95)}{\leq} \frac{\gamma^{2} p^{2} n^{2}}{\mu^{2}(1-\mu) p\left|Y^{\prime}\right|} \stackrel{(71)}{\leq} \mu p\left|X_{1}\right|
$$

vertices $x_{1}^{\prime} \in X_{1}$ satisfy

$$
\operatorname{deg}_{\Gamma}\left(x_{1}, x_{1}^{\prime}, Y^{\prime}\right) \leq(1+\mu) p\left|N_{\Gamma}\left(x_{1}, Y^{\prime}\right)\right|\left|\leq(1+\mu)^{2} p^{2}\right| Y^{\prime} \mid .
$$

(C) for every $\left(x_{1}, x_{1}^{\prime}\right) \in X_{1} \times X_{1}$ satisfying (93), all but at most

$$
\frac{\gamma^{2} p^{4} n^{2}}{\mu^{2} p^{2}\left|N_{B}\left(x_{1}, x_{1}^{\prime}, X_{2}\right)\right|} \stackrel{(93)}{\leq} \frac{\gamma^{2} p^{2} n^{2}}{\mu^{2}(1-\sqrt{\mu}) \varrho^{2}\left|X_{2}\right|} \stackrel{(71)}{\leq} \mu p^{2}|Y|
$$

vertices $y \in Y^{\prime}$ satisfy

$$
\operatorname{deg}_{\Gamma}\left(y, N_{B}\left(x_{1}, x_{1}^{\prime}, X_{2}\right)\right) \leq(1+\mu) p \operatorname{deg}_{B}\left(x_{1}, x_{1}^{\prime}, X_{2}\right) .
$$

For the proof of the upper bound on $t_{2,2}$ we consider the following "exceptional" 5 -tuples $\left(x_{1}, x_{1}^{\prime}, y, x_{2}, x_{2}^{\prime}\right) \in X_{1}^{2} \times Y^{\prime} \times X_{2}^{2}$

$$
\begin{aligned}
& \mathcal{T}_{1}=\left\{\left(x_{1}, x_{1}^{\prime}, y, x_{2}, x_{2}^{\prime}\right):(95) \text { fails for } x_{1} \text { and } x_{2}, x_{2}^{\prime} \in N_{\Gamma}\left(y, X_{2}\right)\right\} \text {, } \\
& \mathcal{T}_{2}=\left\{\left(x_{1}, x_{1}^{\prime}, y, x_{2}, x_{2}^{\prime}\right):(95) \text { holds for } x_{1} \text { but (97) fails for } x_{1}^{\prime}\right. \text {, } \\
& \left.y \in N_{\Gamma}\left(x_{1}, Y^{\prime}\right), \text { and } x_{2}, x_{2}^{\prime} \in N_{\Gamma}\left(y, X_{2}\right)\right\}, \\
& \mathcal{T}_{3}=\left\{\left(x_{1}, x_{1}^{\prime}, y, x_{2}, x_{2}^{\prime}\right): \text { (95) holds for } x_{1},(97) \text { holds for } x_{1}^{\prime}\right. \text {, } \\
& \text { but (93) fails for } \left.\left(x_{1}, x_{1}^{\prime}\right), y \in N_{\Gamma}\left(x_{1}, x_{1}^{\prime}, Y^{\prime}\right) \text {, and } x_{2}, x_{2}^{\prime} \in N_{\Gamma}\left(y, X_{2}\right)\right\} \text {, }
\end{aligned}
$$

and

$$
\mathcal{T}_{4}=\left\{\left(x_{1}, x_{1}^{\prime}, y, x_{2}, x_{2}^{\prime}\right): \text { (95) holds for } x_{1},(97) \text { holds for } x_{1}^{\prime},\right.
$$

(92) holds for $\left(x_{1}, x_{1}^{\prime}\right)$, but (99) fails for $y$ and $\left.x_{2}, x_{2}^{\prime} \in N_{\Gamma}\left(y, X_{2}\right)\right\}$. 
We note that

$$
\begin{aligned}
t_{2,2} \leq\left|\mathcal{T}_{1}\right|+\left|\mathcal{T}_{2}\right|+ & \left|\mathcal{T}_{3}\right|+\left|\mathcal{T}_{4}\right| \\
& +\left|X_{1}\right|^{2} \cdot(1+\mu)^{2} p^{2}\left|Y^{\prime}\right| \cdot(1+\mu)^{2} p^{2}(1+\sqrt{\mu})^{2} \varrho^{4}\left|X_{2}\right|^{2},
\end{aligned}
$$

where the last term stands for the 5-tuples $\left(x_{1}, x_{1}^{\prime}, y, x_{2}, x_{2}^{\prime}\right)$ for which (93), (95), (97), and (99) hold. Since $\left|N_{\Gamma}\left(y, X_{2}\right)\right| \leq(1+\xi) p\left|X_{2}\right|$ for every $y \in Y^{\prime}$ the estimates from (92)-(99) imply

$$
\begin{aligned}
& \left|\mathcal{T}_{1}\right| \stackrel{(94)}{\leq} \mu p^{2}\left|X_{1}\right| \cdot\left|X_{1}\right| \cdot\left|Y^{\prime}\right| \cdot(1+\xi)^{2} p^{2}\left|X_{2}\right|^{2}, \\
& \left|\mathcal{T}_{2}\right| \stackrel{(96)}{\leq}\left|X_{1}\right| \cdot \mu p\left|X_{1}\right| \cdot(1+\mu) p\left|Y^{\prime}\right| \cdot(1+\xi)^{2} p^{2}\left|X_{2}\right|^{2}, \\
& \left|\mathcal{T}_{3}\right| \stackrel{(92)}{\leq} \frac{\sqrt{\mu}}{\varrho^{2}}\left|X_{1}\right|^{2} \cdot(1+\mu)^{2} p^{2}\left|Y^{\prime}\right| \cdot(1+\xi)^{2} p^{2}\left|X_{2}\right|^{2},
\end{aligned}
$$

and

$$
\left|\mathcal{T}_{4}\right| \stackrel{(98)}{\leq}\left|X_{1}\right|^{2} \cdot \mu p^{2}\left|Y^{\prime}\right| \cdot(1+\xi)^{2} p^{2}\left|X_{2}\right|^{2}
$$

Applying these estimates to (100) gives

$$
\begin{aligned}
t_{2,2} \leq & \mu p^{2}\left|X_{1}\right| \cdot\left|X_{1}\right| \cdot\left|Y^{\prime}\right| \cdot(1+\xi)^{2} p^{2}\left|X_{2}\right|^{2} \\
& +\left|X_{1}\right| \cdot \mu p\left|X_{1}\right| \cdot(1+\mu) p\left|Y^{\prime}\right| \cdot(1+\xi)^{2} p^{2}\left|X_{2}\right|^{2} \\
& +\frac{\sqrt{\mu}}{\varrho^{2}}\left|X_{1}\right|^{2} \cdot(1+\mu)^{2} p^{2}\left|Y^{\prime}\right| \cdot(1+\xi)^{2} p^{2}\left|X_{2}\right|^{2} \\
& +\left|X_{1}\right|^{2} \cdot \mu p^{2}\left|Y^{\prime}\right| \cdot(1+\xi)^{2} p^{2}\left|X_{2}\right|^{2} \\
& +\left|X_{1}\right|^{2} \cdot(1+\mu)^{2} p^{2}\left|Y^{\prime}\right| \cdot(1+\mu)^{2} p^{2}(1+\sqrt{\mu})^{2} \varrho^{4}\left|X_{2}\right|^{2} \\
\leq & \left(1+\mu^{\prime}\right) \varrho^{4} p^{4}\left|X_{1}\right|^{2}\left|X_{2}\right|^{2}\left|Y^{\prime}\right|,
\end{aligned}
$$

where the last inequality follows from the choice of $\mu$ from (70), which ensures $\sqrt{\mu}(1+\mu)^{2}(1+\xi)^{2} / \varrho^{2} \leq \mu^{\prime} \varrho^{4} / 5$ and $(1+\mu)^{4}(1+\sqrt{\mu})^{2} \leq 1+\mu^{\prime} / 5$. This concludes the proof of (74).

\section{Concluding Remarks}

In this paper, we prove an extension of the well known theorem of Ruzsa and Szemerédi for subgraphs of $\left(p, \gamma p^{3} n\right)$-bijumbled graphs $\Gamma$ with sufficiently small $\gamma>0$ (see Theorem 3). It would be interesting to find weaker assumptions for the host graph $\Gamma$ which allow one to prove the same result. In particular, one can ask if the jumbledness condition in Theorem 3 can be replaced by $(p, \beta)$-bijumbledness for some $\beta>\gamma p^{3} n$. Our approach taken here is based on an appropriate triangle counting lemma (Lemma 11), for the proof of which we needed $\beta=\gamma p^{3} n$. We note that the triangle counting lemma fails to be true if $\beta=145 p^{2} n$. Indeed, Alon [1] has constructed $(p, \beta)$-bijumbled graphs with $n$ vertices for $p=n^{-1 / 3} / 4$ and $\beta=145 p^{2} n$ which contain no triangle at all. Using a blowup-type construction one obtains $\left(p, 145 p^{2} n\right)$-bijumbled graphs $\Gamma_{p}$ for any $p$ with $n^{-1 / 3} \ll p=o(1)$ which are triangle-free. Since all subgraphs of $\Gamma_{p}$ are triangle-free, the conclusion of Lemma 11 can hold only for $(p, \beta)$-bijumbled graphs with $\beta<145 p^{2} n$. We believe that under the assumption $\beta=o\left(p^{2} n\right)$ Lemma 11 is true. Such a result would imply 
that Theorem 3 holds even if $\gamma p^{3} n$ is replaced by $\gamma p^{2} n$ and the condition on $\lambda(A)$ in Theorem 4 is replaced by $\lambda(A) \leq \gamma|A|^{2} / n$.

In the proof of the triangle counting lemma, we make use of the implication "DISC implies PAIR" (see part ( $a$ ) of Lemma 9), which was established under the condition that $\beta \leq \gamma p^{2} n$. Note that the reverse implication (see Lemma $9(b)$ ) holds under a less restrictive assumption, namely, $\beta \leq \gamma p^{3 / 2} n$. Finding the thresholds for $\beta$ under which these implications are valid remains an open problem.

\section{REFERENCES}

[1] N. Alon, Explicit Ramsey graphs and orthonormal labelings, Electronic Journal of Combinatorics 1 (1994), Research Paper 12, approx. 8 pp. (electronic). 2.1, 6

[2] N. Alon and J. H. Spencer, The probabilistic method, third ed., Wiley-Interscience Series in Discrete Mathematics and Optimization, John Wiley \& Sons Inc., Hoboken, NJ, 2008, With an appendix on the life and work of Paul Erdös. 2.1, 4.2.1, 4.2.4

[3] J. Bourgain, Roth's theorem on progressions revisited, J. Anal. Math. 104 (2008), 155-192. 1

[4] T. C. Brown and J. P. Buhler, A density version of a geometric Ramsey theorem, J. Combin. Theory Ser. A 32 (1982), no. 1, 20-34. 4, 4.2.3

[5] W. G. Brown, P. Erdős, and V. T. Sós, Some extremal problems on r-graphs, New directions in the theory of graphs (Proc. Third Ann Arbor Conf., Univ. Michigan, Ann Arbor, Mich, 1971), Academic Press, New York, 1973, pp. 53-63. 1

[6] F. R. K. Chung and R. L. Graham, Quasi-random subsets of $Z_{n}$, Journal of Combinatorial Theory, Series A 61 (1992), no. 1, 64-86. 2

[7] F. R. K. Chung, R. L. Graham, and R. M. Wilson, Quasi-random graphs, Combinatorica 9 (1989), no. 4, 345-362. 2, 2.3

[8] P. Erdős, M. Goldberg, J. Pach, and J. Spencer, Cutting a graph into two dissimilar halves, J. Graph Theory 12 (1988), no. 1, 121-131. 2.1

[9] P. Erdős and J. Spencer, Imbalances in k-colorations, Networks 1 (1971/72), 379-385. 2.1

[10] J. Fox, A new proof of the triangle removal lemma, preprint. 1

[11] P. Frankl, R. L. Graham, and V. Rödl, On subsets of abelian groups with no 3-term arithmetic progression, J. Combin. Theory Ser. A 45 (1987), no. 1, 157-161. 4

[12] B. Green, Roth's theorem in the primes, Annals of Mathematics. Second Series 161 (2005), no. 3, 1609-1636. 1

[13] B. Green and T. Tao, Restriction theory of the Selberg sieve, with applications, Journal de Théorie des Nombres de Bordeaux 18 (2006), no. 1, 147-182. 2.2

[14] , The primes contain arbitrarily long arithmetic progressions, Annals of Mathematics. Second Series 167 (2008), no. 2, 481-547. 1

[15] Y. Kohayakawa, Szemerédi's regularity lemma for sparse graphs, Foundations of computational mathematics (Rio de Janeiro, 1997), Springer, Berlin, 1997, pp. 216-230. 1, 3

[16] Y. Kohayakawa, T. Luczak, and V. Rödl, Arithmetic progressions of length three in subsets of a random set, Acta Arithmetica 75 (1996), no. 2, 133-163. 1

[17] Y. Kohayakawa and V. Rödl, Szemerédi's regularity lemma and quasi-randomness, Recent advances in algorithms and combinatorics, CMS Books Math./Ouvrages Math. SMC, vol. 11, Springer, New York, 2003, pp. 289-351. 1, 3

[18] M. Krivelevich and B. Sudakov, Pseudo-random graphs, More sets, graphs and numbers, Bolyai Soc. Math. Stud., vol. 15, Springer, Berlin, 2006, pp. 199-262. 2.1, 4.2.3, 4.2.4

[19] V. F. Lev, Progression-free sets in finite abelian groups, J. Number Theory 104 (2004), no. 1, 162-169. 4

[20] R. Meshulam, On subsets of finite abelian groups with no 3-term arithmetic progressions, J. Combin. Theory Ser. A $\mathbf{7 1}$ (1995), no. 1, 168-172. 4

[21] H. H. Nguyen, On distribution of three-term arithmetic progressions in sparse subsets of $\mathbf{F}_{p}^{n}$, arXiv:0905.3890v2, 16 pp., 2009. 1

[22] Y. Peng, V. Rödl, and A. Ruciński, Holes in graphs, Electronic Journal of Combinatorics 9 (2002), no. 1, Research Paper 1, 18 pp. (electronic). 5.3

[23] V. Rödl and M. Schacht, Generalizations of the removal lemma, Combinatorica 29 (2009), no. $4,467-501.1$ 
[24] K. F. Roth, On certain sets of integers, J. London Math. Soc. 28 (1953), 104-109. 1

[25] I. Z. Ruzsa and E. Szemerédi, Triple systems with no six points carrying three triangles, Combinatorics (Proc. Fifth Hungarian Colloq., Keszthely, 1976), Vol. II, Colloq. Math. Soc. János Bolyai, vol. 18, North-Holland, Amsterdam, 1978, pp. 939-945. 1, 1, 4.2.3

[26] M. Schacht, Extremal results for random discrete structures, submitted, 2009, 27 pp. 1

[27] E. Szemerédi, Regular partitions of graphs, Problèmes combinatoires et théorie des graphes (Colloq. Internat. CNRS, Univ. Orsay, Orsay, 1976), Colloq. Internat. CNRS, vol. 260, CNRS, Paris, 1978, pp. 399-401. 2.3

[28] A. Thomason, Pseudorandom graphs, Random graphs '85 (Poznań, 1985), North-Holland Math. Stud., vol. 144, North-Holland, Amsterdam, 1987, pp. 307-331. 2

[29] _ Random graphs, strongly regular graphs and pseudorandom graphs, Surveys in combinatorics 1987 (New Cross, 1987), London Math. Soc. Lecture Note Ser., vol. 123, Cambridge Univ. Press, Cambridge, 1987, pp. 173-195. 2

Instituto de Matemática e Estatística, Universidade de São Paulo, Rua do Matão 1010, 05508-090 São Paulo, Brazil

E-mail address: yoshi@ime.usp.br

Department of Mathematics and Computer Science, Emory University, Atlanta, Ga 30322, USA

E-mail address: rodl@mathcs.emory.edu

Fachbereich Mathematik, Universität Hamburg, Bundesstrasse 55, D-20146 Hamburg, GERMANY

E-mail address: schacht@math.uni-hamburg.de

Department of Mathematics, London School of Economics, Houghton Street, LonDON WC2A 2AE, United Kingdom

E-mail address: J.Skokan@lse.ac.uk 